\title{
Analysis and modeling of broadband airgun data influenced by nonlinear internal waves
}

\author{
Scott D. Frank ${ }^{\mathrm{a})}$ \\ Department of Mathematical Sciences, Rensselaer Polytechnic Institute, 110 8th St., Troy, New York 12180 \\ Mohsen Badiey \\ Ocean Acoustics Laboratory, College of Marine Studies, University of Delaware, Newark, Delaware 19716 \\ James F. Lynch \\ Woods Hole Oceanographic Institution, Woods Hole, Massachusetts 02543 \\ William L. Siegmann \\ Department of Mathematical Sciences, Rensselaer Polytechnic Institute, 110 8th St., Troy, New York 12180
}

(Received 1 June 2004; revised 15 September 2004; accepted 15 September 2004)

\begin{abstract}
To investigate acoustic effects of nonlinear internal waves, the two southwest tracks of the SWARM 95 experiment are considered. An airgun source produced broadband acoustic signals while a packet of large nonlinear internal waves passed between the source and two vertical linear arrays. The broadband data and its frequency range $(10-180 \mathrm{~Hz})$ distinguish this study from previous work. Models are developed for the internal wave environment, the geoacoustic parameters, and the airgun source signature. Parabolic equation simulations demonstrate that observed variations in intensity and wavelet time-frequency plots can be attributed to nonlinear internal waves. Empirical tests are provided of the internal wave-acoustic resonance condition that is the apparent theoretical mechanism responsible for the variations. Peaks of the effective internal wave spectrum are shown to coincide with differences in dominant acoustic wavenumbers comprising the airgun signal. The robustness of these relationships is investigated by simulations for a variety of geoacoustic and nonlinear internal wave model parameters. (c) 2004 Acoustical Society of America.
\end{abstract}

[DOI: $10.1121 / 1.1819499$ ]

PACS numbers: 43.30.Zk, 43.30.Bp, 43.30.Pc [AIT]

Pages: $3404-3422$

\section{INTRODUCTION}

Nonlinear internal waves often occur in coastal areas, including the Yellow Sea ${ }^{1}$ and Mediterranean Sea, ${ }^{2}$ and off both coasts of the United States. ${ }^{3,4}$ They are now known to strongly influence acoustic propagation through them. Variations in acoustic field and travel time caused by nonlinear internal wave fields have been studied in the Barents $\mathrm{Sea}^{5}$ and in the Gulf of Mexico. ${ }^{6}$ Reference 1 proposed that unexpectedly large, frequency-dependent transmission losses observed in the Yellow Sea resulted from strong internal waves. It was suggested that the effect depended on an effective wavenumber of the internal waves and a classical wave-wave interaction phenomena. This leads to a resonance condition relating the internal wave wavelength and differences between the dominant acoustic mode wavenumbers.

Resonant interactions between linear internal waves and acoustic mode amplitudes were previously examined theoretically ${ }^{7}$ in a random media setting. Throughout this paper, we focus mainly on acoustic influences from quasideterministic nonlinear internal waves, as opposed to a random internal wave field. ${ }^{8}$ Fundamental questions arise concerning the ocean applicability of the resonance mechanism. For ex-

\footnotetext{
${ }^{a}$ Currently at the Department of Mathematics, Marist College, 3399 North Rd., Poughkeepsie, New York 12601.

Electronic mail: scott.frank@marist.edu
}

ample, at moderate acoustic frequencies of $200-600 \mathrm{~Hz}$, shallow-water channels have many propagating modes and many possibilities for wavenumber differences that resonate with the internal wave scales. What happens at lower frequencies? The classical resonance mechanism also requires an acoustic diffraction grating with well-defined environmental wavelengths. How are these wavelengths specified in realistic ocean environments, with trains consisting of variable-amplitude, irregularly spaced nonlinear waves? Significant acoustic mode coupling is now known to occur without the resonance mechanism; numerical studies show mode amplitude changes of $400 \mathrm{~Hz}$ signals due to the interaction with a single nonlinear internal wave ${ }^{9}$ and an irregular internal wave packet. ${ }^{10}$ Is the acoustic-nonlinear internal wave resonance condition viable in actual ocean environments?

Simulations support the resonance condition hypothesis as a possible coupling mechanism. For example, in the Yellow Sea study ${ }^{1}$ nonlinear internal wave packets were represented by deterministic sinusoidal waves, so the effective wavenumber was easily specified, and simulations indicated that a resonant interaction could cause the large transmission losses observed. More recently, the role of effective internal wave wavenumbers has been shown for continuous wave (CW) transmissions at $240 \mathrm{~Hz},{ }^{6}$ above $450 \mathrm{~Hz},{ }^{11}$ and for broadband pulses centered at $224 \mathrm{~Hz}$ and above. ${ }^{12,13}$ Nonetheless, we are not aware of combinations of a well-sampled ocean environment, research-quality acoustic data, and a comprehensive modeling study that demonstrates the occur- 
rence and effective operation of the resonance mechanism. Deterministic and random internal wave fields were found by simulations to have distinct effects on acoustic propagation ${ }^{14}$ in an environment like SWARM. A more thorough review of relevant experimental and theoretical investigations has been prepared..$^{15}$ One important development is a formulation ${ }^{16}$ in terms of the horizontal wavenumber spectrum of the nonlinear internal wave packet, as opposed to a single wavenumber. Resonance occurs when internal-wave wavenumber spectrum peaks correspond to differences between acoustic mode wavenumbers.

In the summer of 1995, the Shallow Water Acoustics in Random Media (SWARM) experiment was conducted off the coast of New Jersey ${ }^{17}$ to obtain high quality environmental and acoustic data and study the influences of nonlinear internal waves on acoustic propagation. The main acoustic track in SWARM explored an across shelf geometry, which was perpendicular to the internal wave wavefronts. Broadband signals were also propagated in an along shelf geometry on two southwest tracks during the passage of at least one strong nonlinear internal wave packet. The along and across shelf geometry of these tracks allows the investigation of the azimuthal dependence of acoustic variations caused by nonlinear internal waves. ${ }^{18}$

In this paper we focus on additional observations and detailed modeling for the southwest tracks of the SWARM experiment. The airgun source used along this track produced signals with energy primarily in a low-frequency (10$180 \mathrm{~Hz}$ ) band. This band contrasts with previous studies that focus on CW signals above $200 \mathrm{~Hz}$ and with other broadband simulations $^{12,13,18}$ at higher frequencies. Observed time variations in acoustic measurements, the presence of high quality environmental data, and the passage of a strong and coherent train of nonlinear internal waves provide an exceptional opportunity for testing the applicability of the resonance mechanism. However, low-frequency signals contain relatively few propagating modes and thus provide few opportunities for satisfying the resonance condition. Our study also uses time-frequency analysis to reveal frequencydependent mode amplitude variations resulting from the nonlinear internal waves.

In this paper we also address modeling issues in the context of SWARM that should be relevant to other experiments. It is important for future simulations to develop an environmental model of that site and of the nonlinear internal waves that reproduce observed variations in data. Although questions arise about using weakly nonlinear KortewegdeVries equation solutions to model nonlinear internal waves, ${ }^{19}$ thermal records from SWARM show that these solutions are adequate for modeling spectral and temporal characteristics of the nonlinear wave packet observed. Moreover, the primary acoustic effects seen are reproduced without relying on contributions from a background field of linear internal waves. The level of uncertainty in seabed geoacoustic parameters, especially the upper sediment layers, is detailed for analyzing internal wave effects. An unexpected aspect of the modeling effort was the need to formulate an effective source representation, emphasizing the experimental infor- mation necessary to perform comparisons between data and simulations.

The paper is organized as follows. In Sec. II we provide details of the experimental tracks, including the airgun source and environmental data. In Sec. III we explain environmental observations, notably the occurrence of the nonlinear internal waves and their observed effects on acoustic signals. In Sec. IV we describe the full model of the southwest SWARM tracks obtained from available environmental, source, and acoustic data. In Sec. V we describe parabolic equation results that reproduce observed variations in pulseaveraged intensity and time-frequency behavior. The internal wave-acoustic resonance condition is discussed, and this track provides an effective empirical test of this condition. In Sec. VI, we investigate the sensitivity of pulse-averaged intensity variations to internal wave and geoacoustic model parameters. In Sec. VII we summarize our major findings.

\section{EXPERIMENT DESCRIPTION}

The SWARM experiment was performed off the New Jersey coast in the Mid-Atlantic Bight continental shelf region (Fig. 1, inset). The primary goal of this multiinstitutional experiment was to study effects of nonlinear internal waves on acoustic signals, and a considerable amount of acoustic and environmental data was collected. A full technical overview of SWARM is in Ref. 17. The correlation of acoustic and internal wave data for the southwest tracks can be found in Ref. 18.

In the present work we focus on the southwest experimental tracks shown in Fig. 1. The two vertical linear arrays (VLAs), the primary acoustic receivers for SWARM, are indicated by black circles. The northerly one was a Woods Hole Oceanographic Institution (WHOI) telemetered array deployed in $70.5 \mathrm{~m}$ of water, consisting of 16 hydrophones spaced approximately $3.5 \mathrm{~m}$ apart, with top and bottom phones at depths of 14.9 and $67.5 \mathrm{~m}$. The southerly one, a Naval Research Laboratory (NRL) telemetered array, was located approximately $9 \mathrm{~km}$ to the southeast of the WHOI VLA in $88 \mathrm{~m}$ of water and consisted of 32 elements spaced 2 $\mathrm{m}$ apart with top and bottom phones at 21 and $85 \mathrm{~m}$.

Two thermistor strings (with five thermistors each) at the receiver sites provided "end point" observations of the nonlinear internal waves. The string positions are indicated by white squares in Fig. 1. The first, attached to the WHOI VLA, measured the temperature every $30 \mathrm{~s}$ at depths of 12.5 , $22.5,30.5,50.5$, and $60.5 \mathrm{~m}$. The second, very close to the NRL VLA, took measurements every $60 \mathrm{~s}$ at depths of 19.8 , 39.6, 51.5, 63.5, and $75.5 \mathrm{~m}$. In addition, the R/V Oceanus was near the WHOI array and provided radar images of passing nonlinear internal wave packets. ${ }^{17}$

The R/V Cape Hatteras occupied the SWARM site for six days, 31 July to 5 August, 1995, and was positioned southwest of the two VLAs at the position denoted by the black square in Fig. 1. CTD data collected there also showed the passage of nonlinear internal waves and provided soundspeed profile data. The Cape Hatteras deployed two sources during this period; a J-15 for transmitting LFM sweeps and a 20 in. ${ }^{3}$ Bolt airgun; data from the latter source will be examined here. The airgun had a pulse signature that was found to 


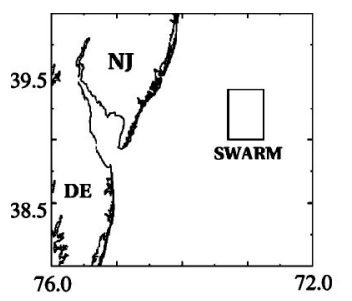

\section{SWARM main track}

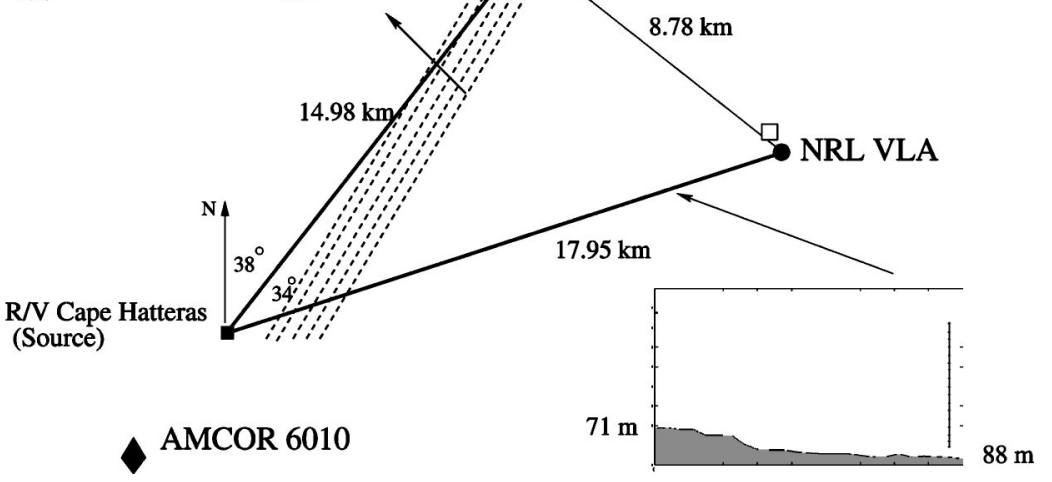

FIG. 1. Geometry of the southwest portion of SWARM experiment. Signals from an airgun at R/V Cape Hatteras (black square) received by WHOI and NRL VLAs (black circles). Thermistor strings (white squares) associated with each array. The AMCOR 6010 site (black diamond) located several $\mathrm{km}$ southwest of the source. Bathymetry of the NRL waveguide declines slightly toward VLA. Two acoustic tracks (dark lines) intersected by an internal soliton packet (dashed lines) observed on 4 August 1995. be quite repeatable, based on measurements at a single monitor hydrophone ${ }^{17,18}$ hung below the source. The airgun produced broadband pulses that had consistently repeatable spectra in the 10-180 Hz band (see Fig. 2 of Ref. 18). These spectra show significant peak frequency components centered near $32 \mathrm{~Hz}$ and several harmonics. The dependable source signature is essential for our subsequent analysis of waveguide-dependent broadband variations.

As shown in Fig. 1 the airgun signals propagated along two different tracks (heavy lines). The track to the WHOI array was about $15 \mathrm{~km}$ long, $70 \mathrm{~m}$ deep, and displayed very flat bathymetry. The NRL track was about $18 \mathrm{~km}$ long; the depth increased from approximately $70 \mathrm{~m}$ at the source to about $88 \mathrm{~m}$ at the VLA. GPS location data estimated the orientation of the WHOI and NRL tracks to be $34^{\circ}$ and $74^{\circ}$ from true north, respectively. The dashed lines in Fig. 1 represent a packet of strong nonlinear internal waves that were observed passing through the experimental region on August 4. The actual packet does not have the exactly linear wavefronts shown schematically here.

The southwest portion of the SWARM experiment is also near the AMCOR 6010 borehole site. Although this site was several km southwest of the Cape Hatteras, it still provides an initial approximation to the geoacoustic properties of the SWARM site. The core location is indicated by the black diamond in Fig. 1.

\section{DATA ANALYSIS}

\section{A. Internal waves}

Several episodes of nonlinear internal wave activity occurred while the R/V Cape Hatteras was on site. ${ }^{17,18}$ Figure 2(a) shows records from the top three WHOI thermistors for August 4. Low-frequency tidal components were removed using a 5 min sliding highpass filter. The passages of several different packets of nonlinear internal waves are visible throughout the day. Figure 2(b) shows a specific group of internal waves that first crossed the WHOI thermistor string at approximately $1800 \mathrm{~h}$ GMT on August 4. By subtracting the DC component from these signals and using the thermistor sampling rate of $1 / 30 \mathrm{~Hz}$, the spectrum of this temperature variability can be calculated. The normalized spectrum in Fig. 2(c) has the strongest peaks at 0.0013 and $0.0024 \mathrm{~Hz}$, which correspond to periods of 12.8 and $6.9 \mathrm{~min}$. This is consistent with the intervals between nonlinear internal waves in the time series of Fig. 2(b).

At 19:01 GMT, while this packet of internal waves was traversing both acoustic tracks, the airgun on the Cape Hat-
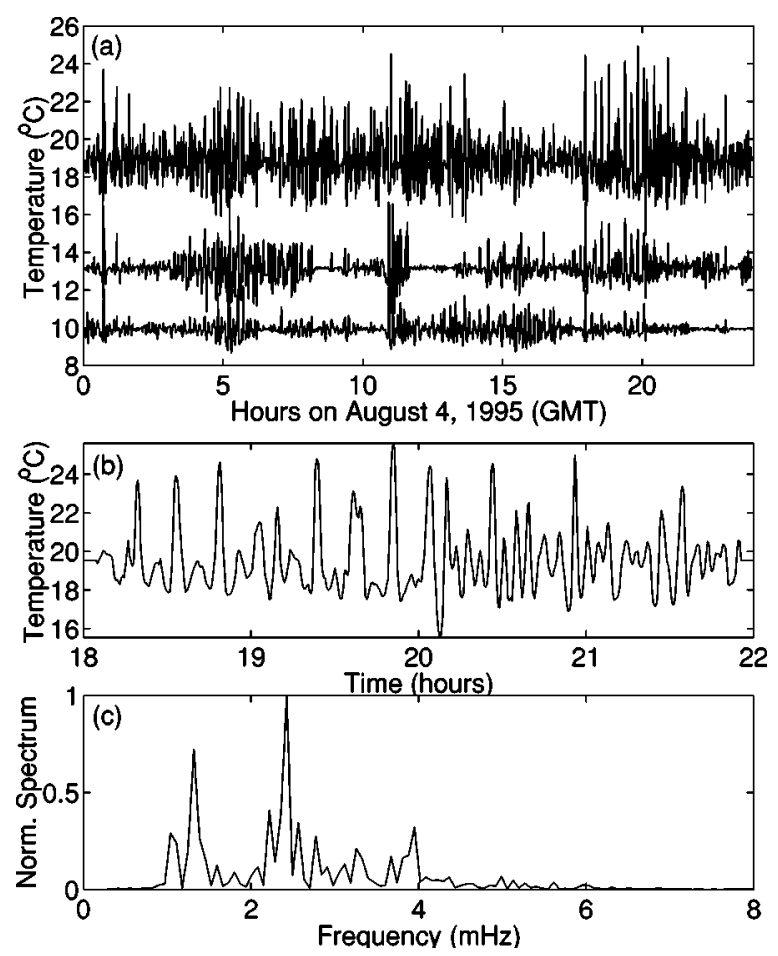

FIG. 2. (a) Records from top three thermistors at WHOI VLA on 4 August 1995. Internal soliton packets visible at all depths. (b) Smoothed record from the top $(12 \mathrm{~m})$ WHOI thermistor between 1800 and 2200 GMT. This packet overlapped acoustic tracks while the airgun fired. (c) The normalized frequency spectrum of the smoothed record in (b) with the DC component removed. Dominant frequency components correspond to periods of 12.82 and $6.94 \mathrm{~min}$. 
teras began firing once per minute. One valuable feature of this data is that the internal wave propagation direction intersects the two acoustic propagation directions at very different angles, as shown in Fig. 1. The bearing of this packet was estimated as $59^{\circ}$ west of north from radar on the R/V Oceanus, which was near the WHOI VLA. Assuming the internal wave crests are linear, the angle between internal wave and acoustic propagation directions is about $49^{\circ}$ along the NRL track and close to $90^{\circ}$ along the other track. At an angle of $90^{\circ}$ the nonlinear internal wave fronts would be parallel to the acoustic track.

\section{B. Acoustics}

\section{Wavelet transform}

The broadband character of the airgun signals allows analyzing changes in their time-frequency behavior after an interaction with nonlinear internal waves. To do this we have used a wavelet transform. ${ }^{20}$ Wavelet transforms have been used, for example, in shallow water for sediment parameter inversion $^{21,22}$ but not for investigating effects of nonlinear internal waves. These transforms help show the frequencydependent variations in arrival times, the strengths of signal components, and the interactions between acoustic modes. A summary of the time-frequency transforms we employed is in Ref. 20.

The time-frequency transform $T_{f}$ of a signal $f(t)$ is given by the integral

$$
T_{f}(u, \xi)=\int_{-\infty}^{\infty} f(t) \phi_{u, \xi}(t) d t,
$$

where $\phi_{u, \xi}(t)$ is a basis function depending on two parameters, $u$ and $\xi$. One familiar example is the Short Time Fourier Transform (STFT), for which the basis functions are sinusoids. For narrow band signals the STFT is effective, but if the signal has many different scales, the STFT can suffer from aliasing or a lack of resolution. For example, a finite time series record has only a few degrees of freedom at lower frequencies, but many at higher frequencies, which prompts the need for scaling. A wavelet basis introduces $\xi$ as a variable scale. We use a Gabor (or Morlet) wavelet that is a Gaussian pulse translated by $u$, scaled by $\xi$, and modulated by chosen frequency $\eta$ :

$$
\phi_{u, \xi}(t ; \eta)=\frac{1}{\sqrt{\xi}} e^{-[(t-u) / \xi]^{2}} e^{i \eta[(t-u) / \xi]} .
$$

When a wavelet basis is used in Eq. (1), the graph of $T_{f}$ is known as a scalogram. Wavelet analysis is not immune to aliasing and interference effects, which depend on the shape of the basis function. However, focusing on scale instead of frequency allows better resolution across a large frequency band, which is desirable since the airgun signal has a reliable frequency content between 10 and $180 \mathrm{~Hz}$.

Figure 3 shows two scalograms of signals from phone 5 (29 m depth) of the NRL VLA. Phone 5 was chosen because its position in the water column is close to a peak in the third acoustic normal mode at $f=64 \mathrm{~Hz}$, and allows a clear observation of first, second, and third mode energy. Each signal is shown in three panels. The top panel is the time domain representation $f(t)$, the right panel is the Fourier spectrum of $f(t)$ over the full interval, and the large panel is the scalogram. The graphs have all been normalized by their maximum values, and the spectrum and scalogram are limited to the repeatable spectrum below $180 \mathrm{~Hz}$. Figure 3(a) shows the signal corresponding to the 1904 GMT airgun shot. Its Fourier transform shows energy peaks near $32 \mathrm{~Hz}$ and its harmonics, with most energy concentrated at 32, 64, and $95 \mathrm{~Hz}$. The scalogram shows the expected modal group velocity structure, ${ }^{23}$ with two modes present at $32 \mathrm{~Hz}$, three at $64 \mathrm{~Hz}$, and at least four at $95 \mathrm{~Hz}$. Mode arrivals at 32, 64, and $95 \mathrm{~Hz}$ are all well separated. Mode interaction causes some energy to travel at different group velocities, which causes energy to appear in the scalogram between otherwise well-defined modes. Some evidence of mode interaction can be seen between the second and third modes of the $120 \mathrm{~Hz}$ band. Figure 3(b), showing the 1910 GMT shot 6 min later, has a Fourier spectrum similar to that from the 1904 shot but with considerably more energy in higher frequencies around 120 and $150 \mathrm{~Hz}$. However, the scalogram reveals even more striking differences between these signals. The modal group velocity curves are still visible at 1910 , but a larger concentration of energy arrives early in higher frequencies. The modal interaction occurring between the first and second modes in the $95 \mathrm{~Hz}$ band is indicated by a strong blending between the corresponding peaks. The mode interaction is also visible above $95 \mathrm{~Hz}$. Shot 1904 has stronger lower frequencies, while shot 1910 has significant high-frequency energy arriving at the start of the pulse. These differences can also be seen in the time series, but not so easily as on the scalograms.

The differences between Fourier transforms and scalograms of these signals are typical in the data from other hydrophones for the entire hour. The variations occur over 12 to 14 minute periods, and indicate the correlation between the nonlinear internal waves and variations in the acoustic signals.

\section{Pulse-averaged intensity}

Variations in pulse-averaged intensity were observed in deconvolved and filtered airgun data. ${ }^{18}$ Since the source was extremely repeatable over $10-180 \mathrm{~Hz}$, we expand the analysis of those variations to the entire reliable band. The decibel time-averaged intensity in $\mathrm{W} / \mathrm{m}^{2}$ of an acoustic pulse $p(t)$ is

$$
I_{T}=10 \log _{10}\left(\frac{1}{T} \int_{0}^{T} \frac{|p(t)|^{2}}{\rho c} d t\right) \mathrm{dB} \text { re: } 1 \mu \mathrm{Pa},
$$

where $T$ is the interval of integration, $\rho$ is water density, and $c$ is an average sound speed. The interval $T$ was chosen to contain a complete pulse and is typically $1.6 \mathrm{~s}$ for NRL VLA data and $1.2 \mathrm{~s}$ for WHOI data.

The quantity $I_{T}$ is calculated for 60 pulses received during the hour beginning on 1901 GMT on August 4. Figure 4(a) shows the results from the WHOI VLA and Fig. 4(b) shows results from the NRL VLA. Multiple variations occur at several $\mathrm{dB}$ amplitudes in both datasets, and the quasiperiods of the variations are from 12 to $14 \mathrm{~min}$. The presence of oscillations with similar periods in both datasets suggests 
(a)
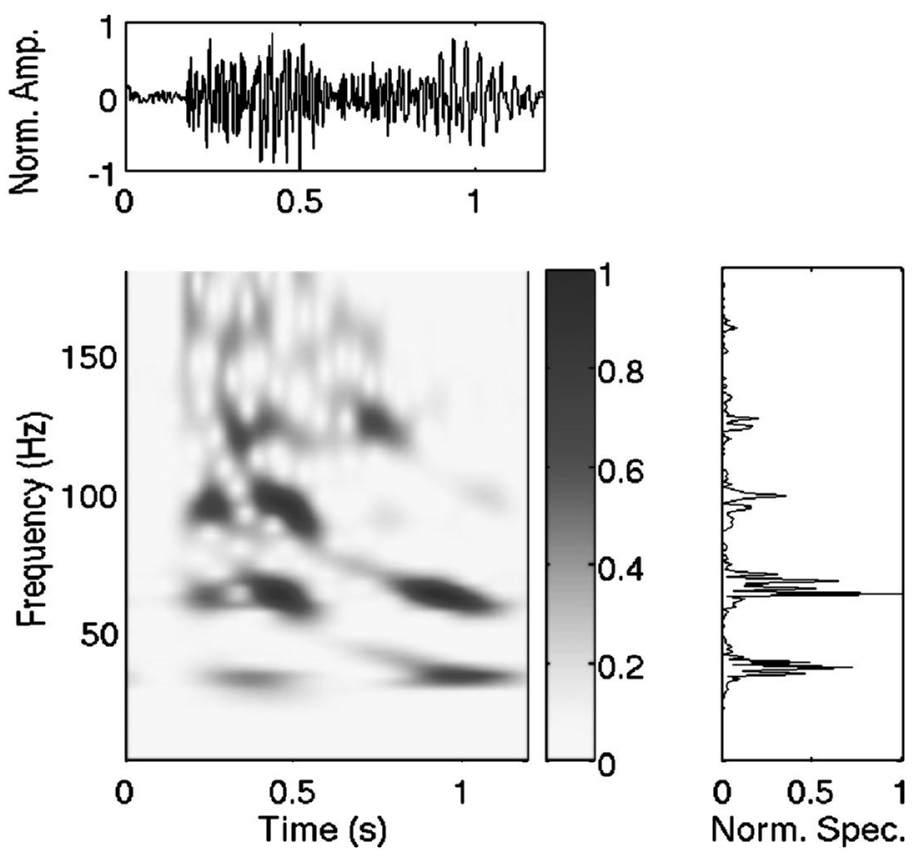

(b)

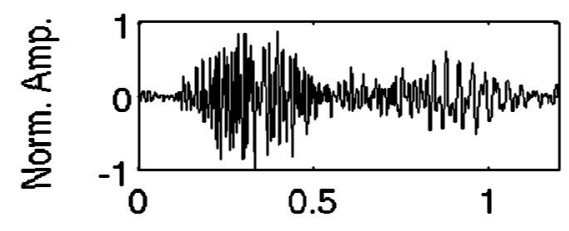

3. A time-frequency analysis of NRL data at $29 \mathrm{~m}$ depth. (a) 1904 GMT shot, received time domain signal (top panel). The Fourier transform (side panel) shows dominant energy peaks at 32, 64, and $95 \mathrm{~Hz}$. The scalogram (color panel) represents a Gabor wavelet analysis of the signal in the top panel. Group velocity curves are visible; two, three, and five modes revealed near 32, 64 , and 95 Hz. (b) 1910 GMT shot (six minutes later). The spectrum shows more high-frequency energy than in (a). The scalogram shows substantial energy arriving early in frequency bands higher than $95 \mathrm{~Hz}$. Evidence of the mode interaction visible, especially at $95 \mathrm{~Hz}$.

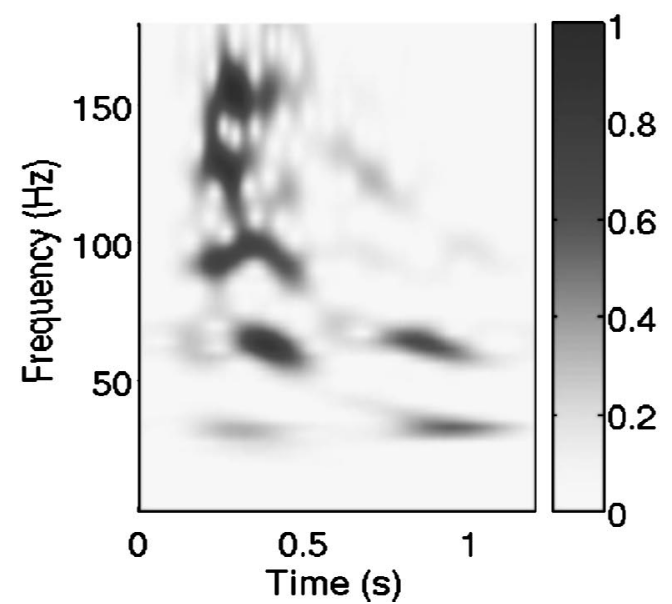

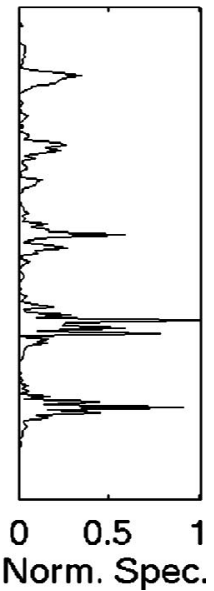

these effects arise from the internal waves that traverse the tracks. The occurrence of these variations is largely independent of receiver depth, although the amplitudes and patterns do show some weak depth dependence. Consequently, a reliable measure of the overall variations can be obtained by examining the depth-averaged $I_{T}$, shown by thick lines in Fig. 4. The quasiperiod of the variations is preserved by the depth average, with amplitudes at the WHOI and NRL VLAs of 5.9 and $3.1 \mathrm{~dB}$, respectively. The difference between these two values suggests that the internal waves have a larger effect on acoustic signals propagating nearly parallel to the internal wave fronts.

\section{MODELING}

Several features of the southwest SWARM tracks are important in order to compare simulations with observations.
The inherent range dependence of the water column due to the internal waves motivates the use of parabolic equation (PE) for calculating the waveguide transfer function. The code RAMGEO ${ }^{24}$ was modified to output the received complex acoustic pressure at selected hydrophone depths, and these values are used to construct the transfer function between 10 and $180 \mathrm{~Hz}$. Broadband pulses are then obtained by standard synthesis methods. ${ }^{25}$ For the remainder of this study, we will focus on modeling the variations observed at the NRL VLA.

\section{A. Internal waves}

Several CTD casts are available from the R/V Cape Hatteras. Sound-speed profiles that were obtained from these are shown in Fig. 2 of Ref. 18. These records show that large internal waves passed under the ship and thus traveled past the source while it was firing. Enough samples were taken to 
(a)

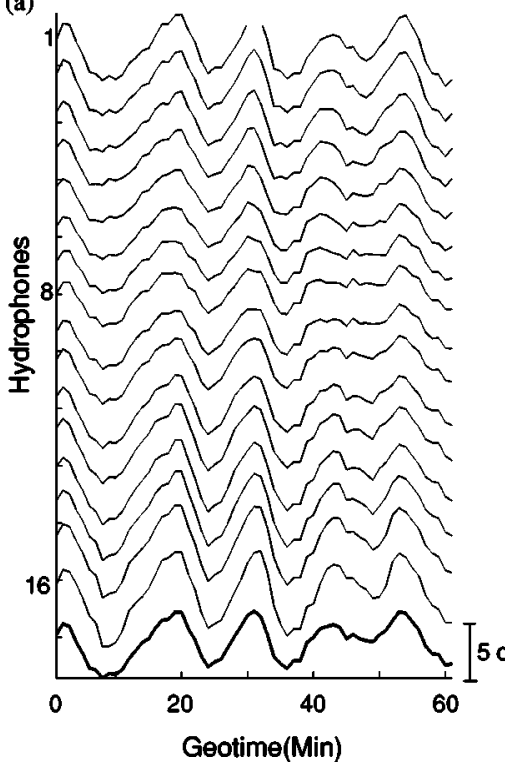

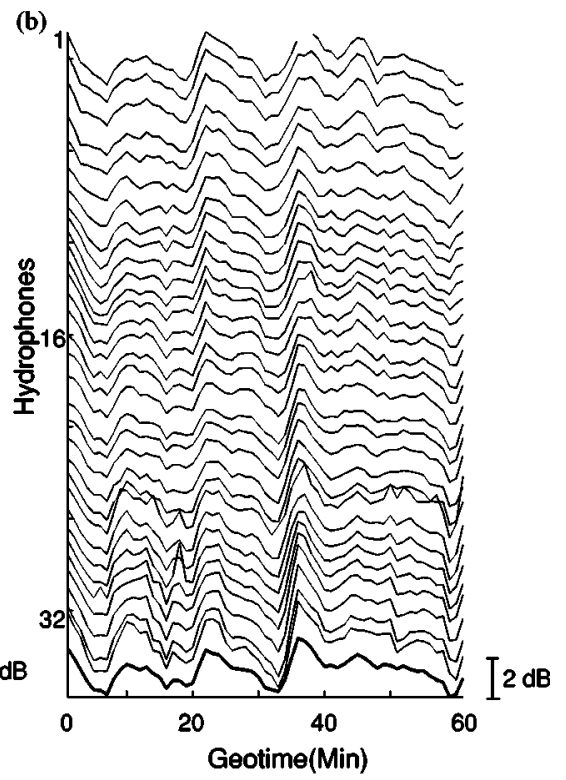

FIG. 4. Intensity $I_{T}$ for each hydrophone at (a) WHOI and (b) NRL VLA versus geotime starting at 1901 GMT. The quasiperiod of variations at each phone is consistent with Fig. 2. Depth-averaged $I_{T}$ (dark curves) shown at the bottom. Note different scales in the range of intensity variations for (a) and (b). obtain a good representation of the average sound-speed profile at the source. This profile is used in the PE simulations. This average profile surely undergoes a modification along the propagation track, but in the absence of data this effect is not incorporated into the environmental model. Only rangedependent variations due to the nonlinear internal waves are included as changes to the average profile.

An early effort to model nonlinear internal waves in shallow water for acoustics work used sinusoidal wave packets. ${ }^{1}$ Since an analysis of weak nonlinear oscillations in a two-layer fluid leads to the Korteweg-deVries (KdV) equation, many authors have used periodic KdV solutions for their models, including the cnoidal ${ }^{6}$ and dnoidal ${ }^{18,26}$ Jacobian elliptical integral solutions. These latter models are more physically correct but not so useful for this study, for two reasons. First, the temporal evolution of an internal wave packet is very slow compared to the passage of an acoustic signal, and second, the full nonlinear internal wave solution is unable to easily incorporate the randomly uneven spacings between wave peaks. ${ }^{15}$ Consequently, a simple sum of nonperiodic $\mathrm{KdV}$ equation solutions is used to represent the horizontal variations due to nonlinear internal waves. Because the sampling rate of the WHOI thermistors is the only experimentally specified parameter, we first regard the packet as a time series:

$$
\eta(t, z)=\Phi_{1}(z) \sum_{n=0}^{5} A_{n} \operatorname{sech}^{2}\left[\frac{2 \pi\left(t-t_{n}\right)}{\tau_{n}}\right] .
$$

Equation (4) represents a train of six $\mathrm{KdV}$ solitons where $t$ represents time, and $t_{n}, A_{n}$, and $\tau_{n}$ represent the peak position (measured from $t=0$ ), amplitude, and width (in seconds) of each soliton. The first internal wave mode is represented by $\Phi_{1}(z)$ and is approximated by a continuous piecewise-linear function. ${ }^{16}$ This model is similar to that used in Ref. 10 . Note that $\tau_{n}$ is not a proper wavelength since the $\operatorname{sech}^{2}$ solutions are not periodic.

We found in our modeling that it is necessary to obtain a reasonable spectral match between data and the modeled internal waves. This has important consequences for the inter- nal wave-acoustic resonance condition in Sec. V, which depends on peaks of the wavenumber spectrum. ${ }^{7,16}$ Due to the remarkably uniform peak spacing and height of the thermal variations in Fig. 2, our model employs evenly spaced internal waves. Figure 5 compares the internal wave packet frequency spectrum to that of the model from Eq. (4) using evenly spaced waves with $\tau_{n}=\tau=11.9$ samples, and $t_{n}$ $=2.3 n \tau$. Standard methods provide the frequency axis from the thermistor sampling rate. The principal features of the two spectra are quite similar. The largest component appears at $f_{i w} \approx 0.0024 \mathrm{~Hz}$, and two smaller components appear at 0.0013 and $0.0040 \mathrm{~Hz}$. The feature match between these two spectra is evidence that our choices of internal wave model and parameters are appropriate for modeling, although these parameters by no means provide a unique representation of the packet. Differences in higher-frequency peaks are caused by slightly uneven spacing between the waves, fluctuations in the water column thermal properties such as the diffuse linear internal wave field, or other effects.

Because of the consistency between the two spectra in Fig. 5, we expect the time series of the nonlinear internal wave packet to be modeled reasonably well. The thermistor

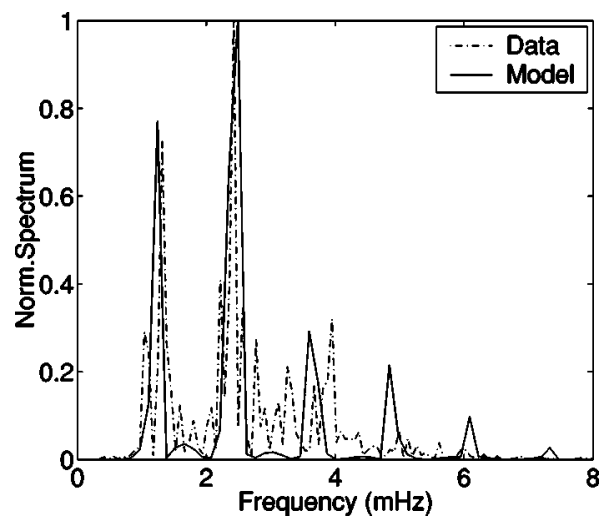

FIG. 5. The dashed curve is a normalized spectrum of an internal wave packet at the top $(12 \mathrm{~m})$ WHOI thermistor reproduced from Fig. 2. The solid curve is the normalized spectrum of the internal wave model in (4). Note the location and amplitude agreement of the dominant frequency components. 

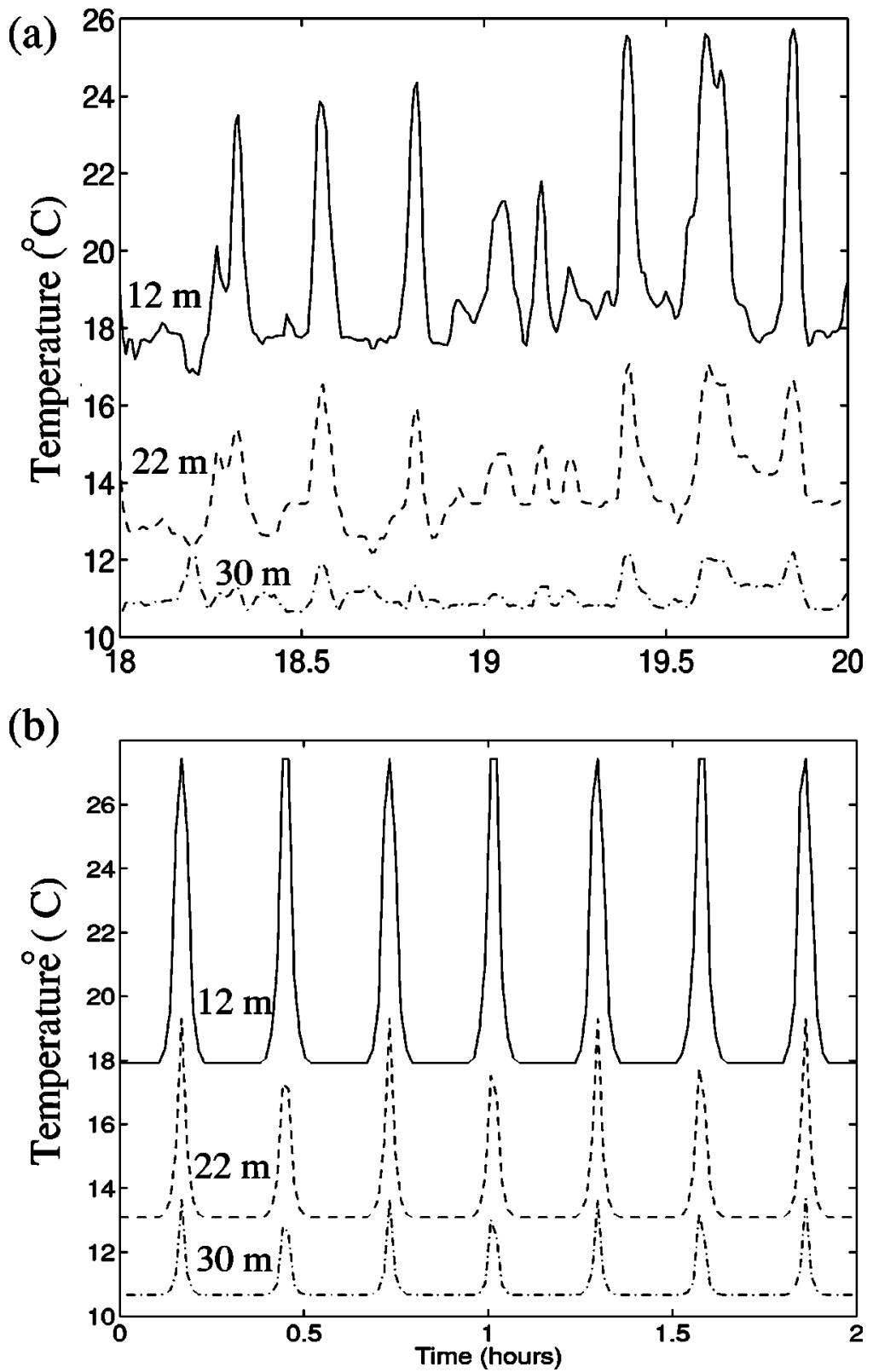

FIG. 6. (a) WHOI thermistor records between 1800 and 2000 GMT on 4 August 1995. The first internal wave mode suppresses the temperature change for increasing depth. Note the regular spacing between oscillations in these records. (b) Simulated thermistor records using the internal wave model in (4) with parameters that generated the spectrum in Fig. 5. sampling rate permits a comparison of the model time series to data. Figure 6(a) shows three thermistor records of the type in Fig. 2(b) that focus on the nonlinear internal wave packet passing the WHOI thermistor string between 1800 and 2000 GMT. As the internal waves pass any location, they force warm water into the lower colder layer. The amount of temperature change decreases with depth, as seen by comparing the solid curve (12 $\mathrm{m}$ depth) in Fig. 6(a) to the dashed $(22 \mathrm{~m})$ and dashed-dotted $(30 \mathrm{~m})$ curves. From the model spectrum of the internal wave packet, the associated frequency domain representation is inverse Fourier transformed to obtain the time series, shown in Fig. 6(b). The first internal gravity wave mode used in the model expresses thermal variations as depth increases as in the data. Uniform peak spacing and height is reproduced in the model, as expected, although different parameters could be selected for a more detailed pattern match. Because of the relatively high symmetry in the data, we chose parameters for a symmetric model and thus a classical diffraction grating. Since diffrac- tion gratings cause incident waves to break into bands of maxima and minima behind the grating, ${ }^{27}$ this phenomenon provides an interpretation of the variations observed in our particular data. We emphasize that our conclusions do not show that either uniform spacing between waves or identical amplitudes is essential to model the observed spectrum. Indeed it has been shown that even a single wave can cause acoustic mode coupling, ${ }^{9}$ although more than one wave is evidently necessary for a reasonable spectral match to this data.

The acoustic effects of linear internal wave field components of the internal wave field ${ }^{28}$ are often distinct from the effects caused by nonlinear internal waves. ${ }^{14,29}$ Calculations were performed to confirm that the environment under consideration is consistent with that conclusion, but these results are not shown here. Satellite images of coastal nonlinear internal waves ${ }^{4,17}$ indicate that assuming essentially linear wavefronts in this region is reasonable. However, this as- 

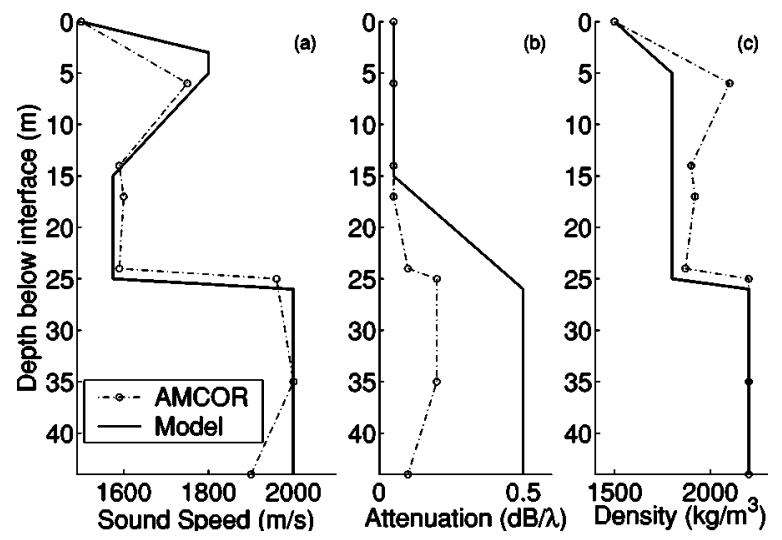

FIG. 7. AMCOR 6010 data (dashed curves with circles) and model geoacoustic profiles (solid curves) for (a) sound speed $(\mathrm{m} / \mathrm{s})$, (b) attenuation $(\mathrm{dB} / \lambda)$, and $(\mathrm{c})$ density $\left(\mathrm{kg} / \mathrm{m}^{3}\right)$. Principal characteristics including upper sediment layer, shallow reflector, low sound speed waveguide, and strong reflector correspond. The large attenuation values in the model account for elastic effects.

sumption introduces uncertainty into any environmental model of the SWARM region.

\section{B. Geoacoustics}

The NRL track bathymetry is slightly sloping from 71 to $86 \mathrm{~m}$ (inset, Fig. 1) according to depth values that were obtained from the National Geophysical Data Center. ${ }^{30}$ We assume that sediment layer interfaces in the bottom follow the bathymetry, so that new layers are not revealed as the water depth increases. A PE implementation that handles bottom layers in this way, RAMGEO, ${ }^{24}$ was modified to output complex pressure at given hydrophone depths and then used to calculate the waveguide transfer function.

Data from the AMCOR 6010 site (see Fig. 1) has significant features that we preserved in our bottom model. Core profiles of compressional sound speed, attenuation, and density are shown by dashed lines in Fig. 7, where small circles represent the actual data points. ${ }^{17}$ One feature is a shallow reflector within $10 \mathrm{~m}$ of the water-sediment interface. A deep layer of dense fast material appears about $25 \mathrm{~m}$ from the water-sediment interface, with a channel of softer sediment between these two reflectors. The presence of the deep strong reflector has been well documented for this region. ${ }^{31}$ We considered a variety of candidate bottom profiles that preserved these features and were numerically "close" to the data profiles. To evaluate a candidate bottom model, PE simulations were performed and the received pulses filtered using an order 10 noncausal Butterworth filter. ${ }^{32}$ This filter was applied with $10 \mathrm{~Hz}$ bandwidth and center frequencies chosen at the dominant airgun signal frequencies (source details are shown in Sec. IV C). The goal was reasonable visual agreement between relative modal amplitudes and arrival times in data and simulations. This procedure for selecting geoacoustic profiles is similar to that used elsewhere, ${ }^{33,34}$ and no strong claim can be made about the faithfulness of the result to the actual bottom profile (for example, the geoacoustic range dependence is not modeled) or about its uniqueness. Bottom profile inversions at the SWARM site are being performed by others. ${ }^{35}$
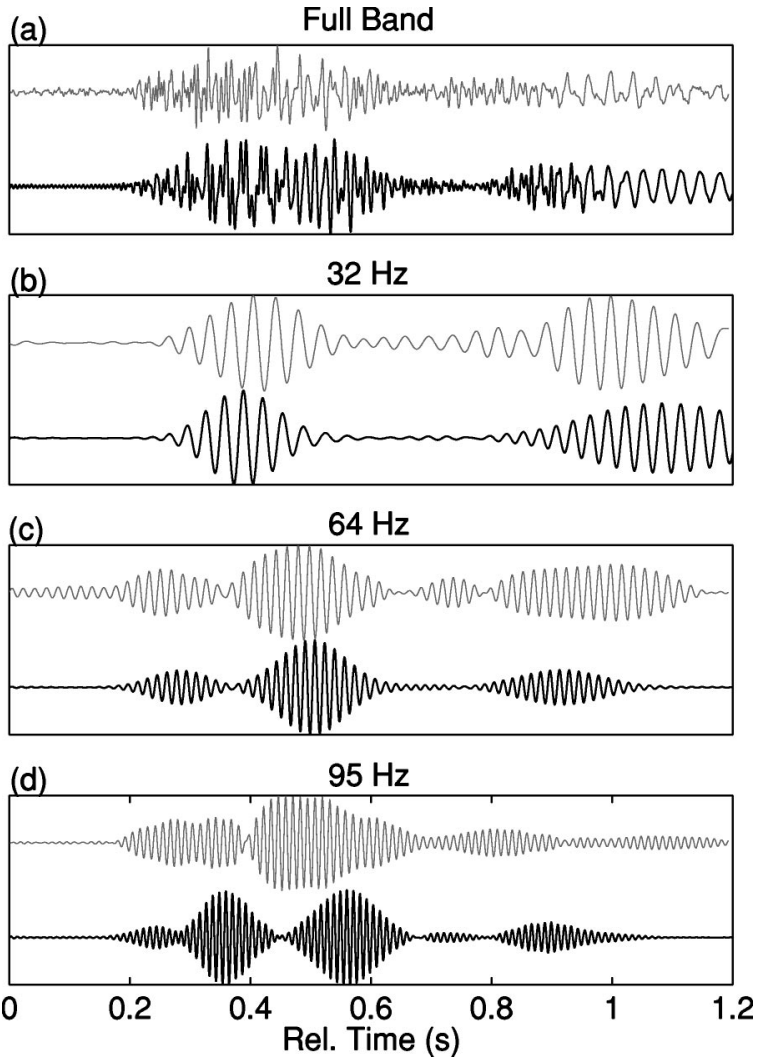

FIG. 8. Comparisons showing the similarity between modal characteristics (occurrence, dispersion, and strength) in pulse data (light upper curves) and simulations (heavy lower curves). (a) Full frequency band. (b) Light (and heavy) curve results from applying a $10 \mathrm{~Hz}$ bandwidth Butterworth filter centered at $32 \mathrm{~Hz}$ to a light (and heavy) broadband signal in (a). (c) The same, but with the filter centered at $64 \mathrm{~Hz}$. (d) The same, but with the filter centered at $95 \mathrm{~Hz}$

The selected bottom profiles are shown by solid lines in Fig. 7. The upper reflective layer, slow channel, and strong reflector are preserved in Fig. 7(a). Since the strong reflector is probably hard material that supports shear waves, the increased attenuation in Fig. 7(b) is expected since elastic effects are not explicitly included. ${ }^{36}$ Figure 8(a) compares a typical NRL data shot (light curve) with a 10-180 Hz fullband simulation (dark curve) at $29 \mathrm{~m}$ depth using this bottom. Figures 8(b), 8(c), and 8(d) compare Butterworth filtered data (light curves) and simulations (dark curves) in 32, 64, and $95 \mathrm{~Hz}$ bands of the curves in Fig. 8(a). Relative amplitude and arrival times of two modes at $32 \mathrm{~Hz}$ show good agreement. In the $64 \mathrm{~Hz}$ band, the first two modes are well reproduced, while the third is present but smaller than in data. In the $95 \mathrm{~Hz}$ band, the large initial pulse in the simulation represents a combination of the first and second modes, and the presence and arrival of higher modes show good agreement with data. Results at other depths show similar agreement, and $29 \mathrm{~m}$ is displayed to show as many modes as possible. The overall agreement is good considering the geoacoustic parameter uncertainty and neglected effects such as bottom elasticity and profile range dependence.

\section{Source model}

Broadband simulation is usually performed by calculating continuous wave solutions at many discrete frequencies 
in bands of interest to obtain waveguide transfer functions. ${ }^{25}$ The transfer function and the Fourier transform of the source signature are used to obtain the final broadband pulse. Thus, the accuracy of broadband simulations depends on a reliable frequency-domain representation of the source signal. The available source monitor is critical for assuring that the airgun produced repeatable signals, which means that an average of recorded source signatures deviates very little from an individual source signature over the frequency band. However, this monitor hydrophone suffered from apparent contamination in its output, which we explain next.

Figure 9 illustrates inconsistencies between the frequency spectrum of the monitor signal and received VLA data. Figure 9(a) shows a sample pulse recording from hydrophone $5(29 \mathrm{~m})$ of the NRL array at 1910 GMT. Figure 9(b) shows the amplitude-normalized spectrum of this data. Note the gradual decrease in the energy in the second and higher harmonics and that the energy in the first harmonic is slightly higher than in the fundamental. This spectral distribution is typical of all data from both the NRL and WHOI arrays. Consequently, we believe it represents the roughly "correct" distribution of source energy propagated down each waveguide. For comparison, the average of the signals received at the monitor hydrophone is shown in Fig. 9(c), and its normalized spectrum in Fig. 9(d). This spectrum shows significantly more energy in the fundamental band around $32 \mathrm{~Hz}$ than in any other. Peaks occur at 64, 95, and $150 \mathrm{~Hz}$, but the largest of these is less than one-sixth the amplitude of the $32 \mathrm{~Hz}$ peak. The substantially different energy distribution in Fig. 9(d) from that in Fig. 9(b) is suspicious. If the true source spectrum looks like Fig. 9(d) then significantly more low-frequency energy than highfrequency energy would appear in Fig. 9(b). There is actually proportionally more high-frequency energy, Fig. 9(b) - the opposite of what is expected from standard medium attenuation or scattering effects.

Confirmation of the difficulty is provided by Fig. 9(e), which shows the broadband time series at the NRL VLA simulated using this source and previously described bathymetry, water sound speed, and geoacoustic profiles. The strong presence of the $32 \mathrm{~Hz}$ band is suggested by the character of the time series. The normalized spectrum shown in Fig. 9(f) verifies that the frequency composition of the monitor signal in Fig. 9(c) is preserved by the waveguide, as expected in the absence of significant frequency-dependent effects.

The cause of the mismatch between data in Figs. 9(a), 9(b) and the simulations in Figs. 9(e), 9(f) is apparently from signal corruption at the monitoring phone by echoes off the surface and seafloor interfaces. These echoes are not propagated down the waveguide, so a source representation that includes them will cause inaccurate broadband modeling. We next show that, with this particular configuration of the airgun in the waveguide, the echoes lead to abnormal amplification of key frequency components. Suppose two sources are in a homogeneous environment. The first source is $1 \mathrm{~m}$ above a receiver (which represents the monitor), and the second is at a distance above the receiver $(25 \mathrm{~m}$ for the airgun at $12 \mathrm{~m}$ ) that corresponds to the surface echo. At a given time,
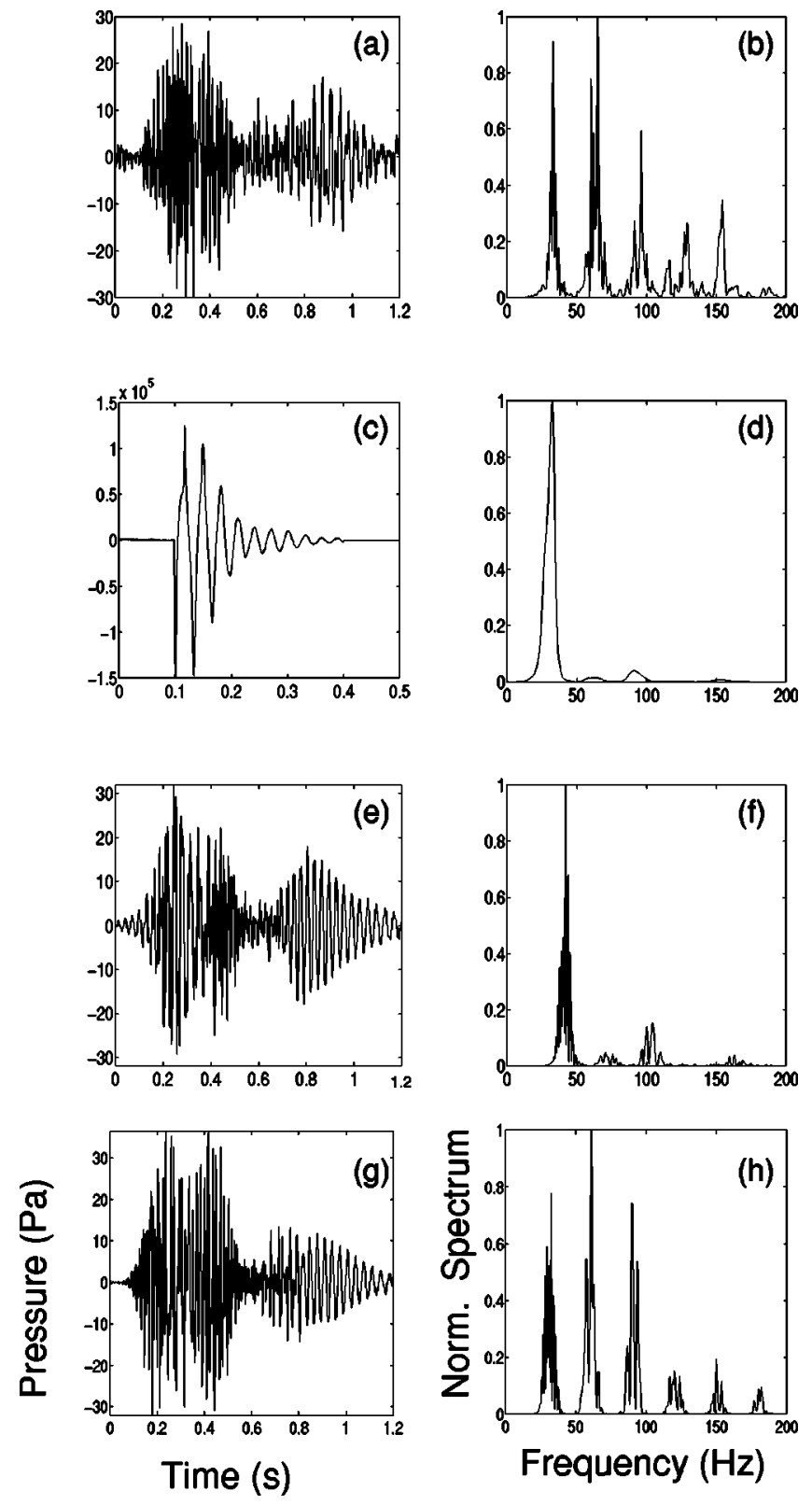

FIG. 9. An illustration of source modeling issues. (a) Sample NRL data pressure $(\mathrm{Pa})$ data signal at $29 \mathrm{~m}$; and (b) its normalized spectrum illustrates a gradual decrease in the energy level of higher-frequency harmonics. (c) The pressure obtained by averaging signals received at a source monitoring hydrophone. (d) Normalized spectrum of (c) contains a relatively large 32 $\mathrm{Hz}$ component. (e) The simulated signal using the source in (c), and (f) its normalized spectrum. Since the source frequency composition is essentially preserved by a waveguide, contamination has occurred in (c). (g) The simulated signal for the same waveguide environment as (e), but the source representation is Gaussian pulses in the frequency domain, and (h) its normalized spectrum. The spectral character matches (b) well.

both sources emit the same signal $f(t)$, for which $\widetilde{F}(z)$ is the $z$-transform from sampling $f(t)$ at an interval $T$. If $g(t)$ is the system output, its $z$-transform is

$$
\widetilde{G}(z)=\widetilde{F}(z)\left(1+z^{-k}\right) \tilde{H}(z),
$$

where $\tilde{H}(z)$ is the transfer function for the homogeneous environment and $z^{-k} \tilde{H}(z)$ is the ( $k$ sampling unit) delayed version of $\tilde{H}(z)$ corresponding to the second source. Range dependence everywhere has been ignored because of the 
small distances involved. Equation (5) shows that the received signal is affected by additional poles at the $k$ th roots of unity, which will amplify certain frequencies. We substitute $e^{i \omega T}$ for $z$ and solve for $\omega$, so that amplification is expected at

$$
\omega_{l}=\frac{\pi}{k T}(1+2 l) \Rightarrow f_{l}=\frac{1}{2 k T}(1+2 l), \quad l=0,1,2, \ldots,
$$

where $k T$ is the total delay between the arrival of the first and second signals. With our configuration, $k T \approx 0.0167 \mathrm{~s}$, and hence $f_{0} \approx 30.8 \mathrm{~Hz}$, so the fundamental in the source signature suffers substantial amplification from the surface echo return. Moreover, for $l=1$ (and $l=2$ ) the echo frequency is about $90 \mathrm{~Hz}$ (and $150 \mathrm{~Hz}$ ), and thus anomalous amplification occurs in both frequency bands in Fig. 9(d), although they are weaker than the echo near $32 \mathrm{~Hz}$. The conclusion is that signals recorded by the source hydrophone, while valuable for establishing the repeatability of the airgun cannot be used in modeling broadband propagation.

Such echoes or any unwanted frequency components can often be removed from a signal by standard filtering techniques. ${ }^{32}$ However, when the corruption occurs in the same frequency bands as the signal of interest, it is not always possible to discriminate between the two receptions. One method is deconvolution techniques to estimate the impulse response of the waveguide. ${ }^{18}$ These techniques require signal processing and assumptions that are not easily justified in this case. However, the repeatability of the source signature suggests that variations in the data are due to the environment and not the airgun source. Thus, we construct a model source signature that preserves the impulsive nature of the airgun, with frequency components, amplitudes, and bandwidths selected so reasonable agreement is obtained between VLA data and broadband simulations.

Since the frequency components in the received data signals are robust, we model the source by summing modulated Gaussian pulses in the frequency domain. The widths and locations of these pulses are chosen to match the dominant features in the spectra of the data from the NRL VLA. With a bandwidth of $2 \sqrt{24 \pi}=17.34 \mathrm{~Hz}$, a model in the frequency domain is

$$
S(\omega)=A \sum_{n=1}^{N} r^{n-1}\left[e^{-\left(\omega-2 \pi q_{n}\right)^{2} / 24 \pi}+e^{-\left(\omega+2 \pi q_{n}\right)^{2} / 24 \pi}\right],
$$

where $A$ is the amplitude, $r$ is a number less than one that governs successive amplitudes of the harmonics, and $q_{n}$ are frequency peak locations. To correspond with the experiment, peaks are chosen at $32 \mathrm{~Hz}$ and its harmonics. The harmonic frequencies need to be reduced by the observed factor of 15/16, because airgun signals are damped by surrounding water pressure (analogous to attenuation in oscillatory systems reducing a frequency to a quasifrequency), so the frequency peaks $q_{n}=32(15 / 16) n \mathrm{~Hz}$ for $n=1,2,3, \ldots$. While other parameters were obtained from the monitor signal, the selection $r=0.65$ is based on energy distributions at the VLA. The impulsive nature of the airgun signal is preserved, although with a quite different character than the monitor signal. ${ }^{37}$
TABLE I. Three strong peaks appear in the WHOI thermistor data frequency spectrum (Fig. 5). An internal wave speed of $0.42 \mathrm{~m} / \mathrm{s}$ gives wavenumbers $\kappa_{i w}$ and wavelengths $\lambda_{i w}$ along the direction of internal wave propagation. The right two columns show values projected onto the NRL track.

\begin{tabular}{lccccc}
\hline \hline \multicolumn{2}{l}{ WHOI thermistor data } & & & \multicolumn{2}{c}{ NRL track parameters } \\
\cline { 1 - 2 } \cline { 5 - 6 } \cline { 5 - 6 }$(\mathrm{Hz})$ & $\lambda_{i w}(\mathrm{~m})$ & $\kappa_{i w}(\mathrm{rad} / \mathrm{m})$ & & $\lambda_{\mathrm{NRL}}(\mathrm{m})$ & $\kappa_{\mathrm{NRL}}(\mathrm{rad} / \mathrm{m})$ \\
\hline 0.00132 & 319 & 0.0197 & & 450 & 0.0140 \\
0.00243 & 173 & 0.0364 & & 244 & 0.0257 \\
0.00395 & 106 & 0.0591 & & 150 & 0.0418 \\
\hline \hline
\end{tabular}

Figure $9(\mathrm{~g})$ shows a simulated time series using the model source. The spectrum of this time series is shown in Fig. 9(h) and clearly better matches the data. All subsequent simulations in this study are calculated using the source model of Eq. (7).

\section{COMPUTATIONAL RESULTS}

Using the environmental and source models from Sec. IV, broadband PE simulations are compared with one hour of airgun data. To obtain the internal wave model in a standard coordinate system of range $r$ and depth $z$, we rewrite Eq. (4) for symmetric waves as

$$
\eta(r, z)=\Phi_{1}(z) \sum_{n=0}^{5} A_{n} \operatorname{sech}^{2}\left[\frac{2 \pi\left(r-r_{n}+v_{n} t\right)}{\Lambda_{n}}\right],
$$

where $v_{n}=v_{i w}$ is a (uniform) internal wave speed, $A_{n}=A$ $=13 \mathrm{~m}, \Lambda_{n}=\Lambda$ is a uniform internal wave width, $r_{n}$ $=s n \Lambda$, and $s$ is called the spacing multiple. Varying $v_{n}$ for each wave would introduce packet dispersion and was judged unnecessary for the current study. For models involving solutions of the $\mathrm{KdV}$ equation, $v_{i w}$ can be estimated from environmental parameters as in Ref. 18. Here we choose $v_{i w}=0.42 \mathrm{~m} / \mathrm{s}$ because when projected onto the NRL acoustic track (discussed below) we obtain $v_{\mathrm{NRL}}=0.6 \mathrm{~m} / \mathrm{s}$, which provides a consistent match with the period of acoustic $I_{T}$ variations in Fig. 4 and is also close to the $0.579 \mathrm{~m} / \mathrm{s}$ value in Ref. 18 for a similar packet of waves.

Column 1 of Table I contains the frequency locations of peaks in the spectrum of thermal data shown in Fig. 2(c). Values for wavelengths $\lambda_{i w}=v_{i w} / f_{i w}$ associated with these frequencies are shown in column 2 , and column 3 shows wavenumbers $\kappa_{i w}=2 \pi / \lambda_{i w}$. To obtain internal wave parameters to model the NRL track, values in columns 2 and 3 are projected using an incidence angle estimate of $45^{\circ}$. Column 4 contains projected column 2 values $\lambda_{\mathrm{NRL}}=\lambda_{i w} / \cos \left(45^{\circ}\right)$, and column 5 shows $\kappa_{\mathrm{NRL}}=2 \pi / \lambda_{\mathrm{NRL}}$. The location of the lowest frequency spectral peak in Fig. 5 corresponds to the periodic distance between evenly spaced nonlinear internal waves. Thus, the nonlinear internal wave width $\Lambda$ in Eq. (8) can be found using $r_{n}=s \Lambda_{\mathrm{NRL}}=\lambda_{\mathrm{NRL}}$. Since $s=2.3$ from Sec. IV A, $\Lambda_{\mathrm{NRL}}=450 / 2.3=195 \mathrm{~m}$.

The projected packet model is propagated toward the source for 60 "minutes" of geotime and a PE simulation is performed for each minute. Results at $29 \mathrm{~m}$ using the experimental bathymetry, water sound speed profiles at the source, and the bottom profiles shown in Fig. 7 are compared to data 
(a)
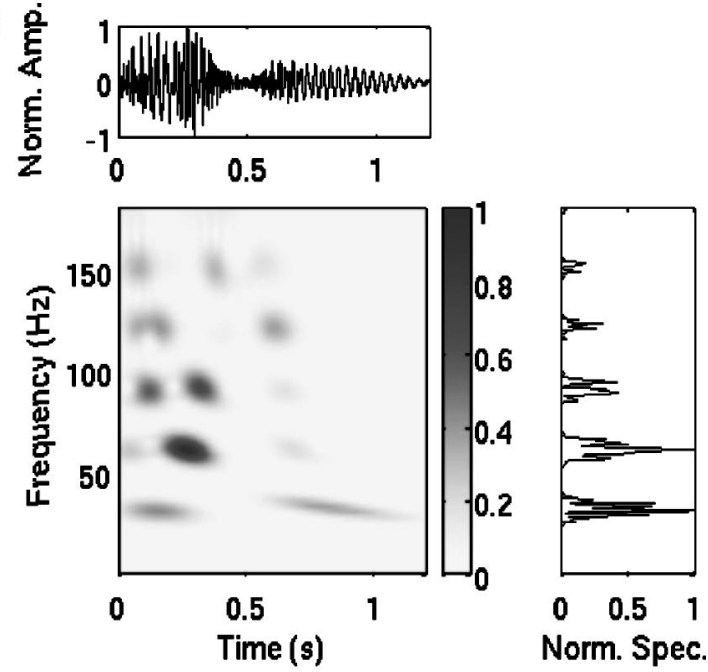

(b)
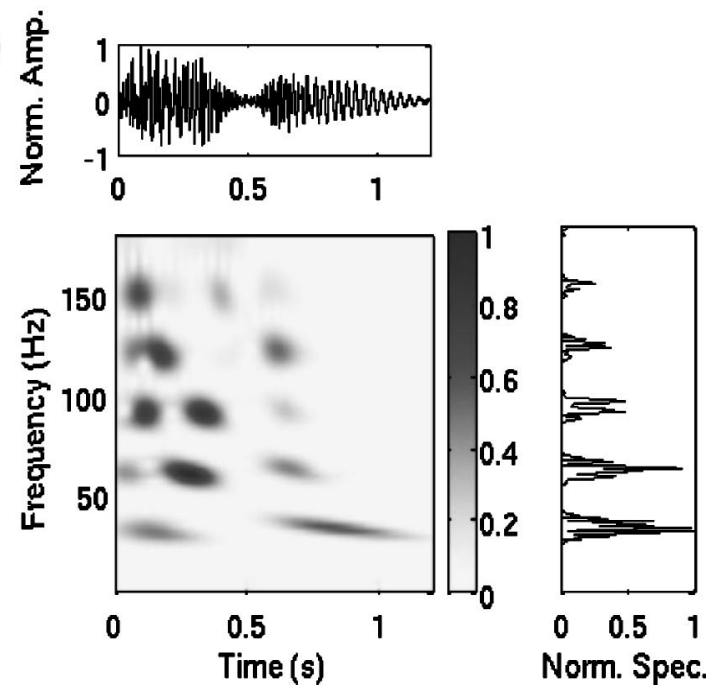

FIG. 10. A Gabor wavelet analysis of broadband signals from PE simulations. The same panels as in Fig. 3. (a) Simulation minute 12 concentrates acoustic energy near $32 \mathrm{~Hz}$ and its harmonics. (b) Simulation minute 18 (6 geotime minutes later) shows an increased higher-frequency content and variations in mode strengths.

shot 1910 GMT in Fig. 8. Note that modes in bands around 32,64 , and $95 \mathrm{~Hz}$ are clearly present (and will thus be able to couple) and that their arrivals are consistent with data. Small discrepancies should not significantly affect scalograms or pulse-averaged intensity.

We next examine scalograms of the simulated signals. Similarity of group velocity curves and modal excitation between the data and simulations is also a good check of the broadband synthesis method. Figure 10 shows two signals that are $6 \mathrm{~min}$ apart at $29 \mathrm{~m}$ depth. Behavior comparable to that in the data scalograms in Fig. 3 is observable. Figure 10(a) shows simulation minute 12 . The time domain pulse suggests, and its Fourier transform shows, significant energy peaks in expected frequency bands. Similar to Fig. 3(a), most signal energy arrives in the second mode of the $64 \mathrm{~Hz}$ band, while well-separated arrivals appear in the 95 and $120 \mathrm{~Hz}$ bands. Two modes appear at $32 \mathrm{~Hz}$, three modes at 64 and 95 $\mathrm{Hz}$, and up to four modes in bands above $95 \mathrm{~Hz}$, consistent with Fig. 3(a). Figure 10(b) shows simulation minute 18 and notable differences from the minute 12 signal. Acoustic en-

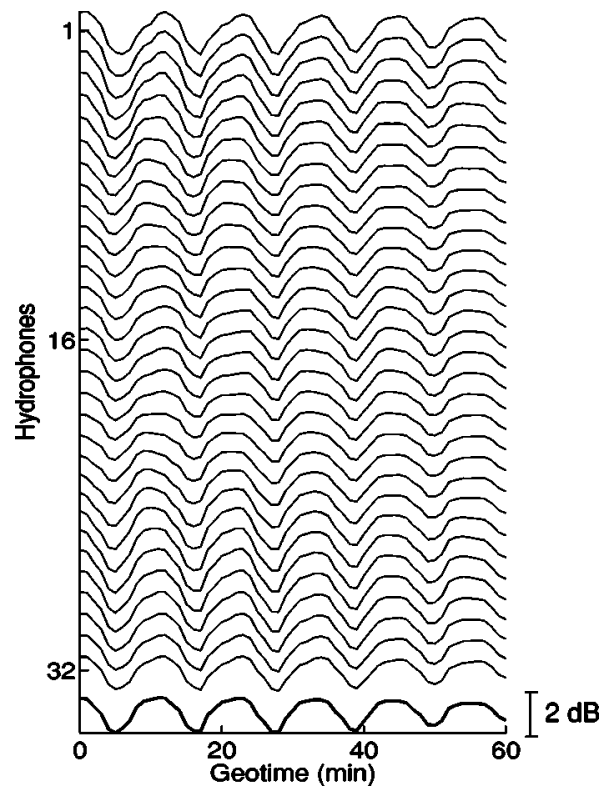

FIG. 11. Intensity $I_{T}$ at each hydrophone for $60 \mathrm{~min}$ traversal of an internal soliton packet past the source. Variations occur clearly at all 32 NRL hydrophones, and the dynamic range of depth-averaged intensity variations closely matches observations (Fig. 4).

ergy has been distributed to the third mode of the $64 \mathrm{~Hz}$ band and into higher-frequency bands. This is the same redistribution as is evident between the data scalograms in Fig. 3. Modes at $95 \mathrm{~Hz}$ and above are not as well separated as at minute 12 , which again is similar to the data. The features are quasiperiodic in the computations with time scales close to those in the data.

Figure 11 shows pulse-averaged intensity $I_{T}$ at each hydrophone of the NRL VLA for the simulation parameters above in a plot analogous to Fig. 4(b). A very close match between simulations and data is evident for the first $30 \mathrm{~min}$. Approximately 3.5 periods occur in the calculations, similar to variations in the data. In the second $30 \mathrm{~min}$, comparably strong variations occur in the data, but their period is longer and does not match the computations. This discrepancy is likely caused by evolution of the nonlinear internal wave packet and its deviations from the model. The overall depth dependence of the calculated $I_{T}$ is very similar to that of the NRL data, with slightly larger oscillations at the top and bottom of the array. The depth-averaged amplitude is very close to that of the data.

Figure 12 displays depth-averaged $I_{T}$ calculations for full-band and bandpass filtered data (solid curves) and computations (dashed-dotted curves). Figure 12(a) shows a very good match between data and computed depth-averaged $I_{T}$. Note a strong amplitude and phase correlation between data and computations for the first $30 \mathrm{~min}$ of geotime. Data variations have maximum amplitude slightly more than $2 \mathrm{~dB}$ for this time period, while the computations have $1.65 \mathrm{~dB}$. The location of intensity maxima and minima is consistent with data, suggesting that the speed estimate is appropriate. Figures 12(b), 12(c), and 12(d) show depth-averaged $I_{T}$ for order-10 Butterworth filtered signals with a $10 \mathrm{~Hz}$ bandwidth and center frequencies of 32, 64, and $95 \mathrm{~Hz}$. At $32 \mathrm{~Hz}$ the data are somewhat noisy, although one large intensity mini- 

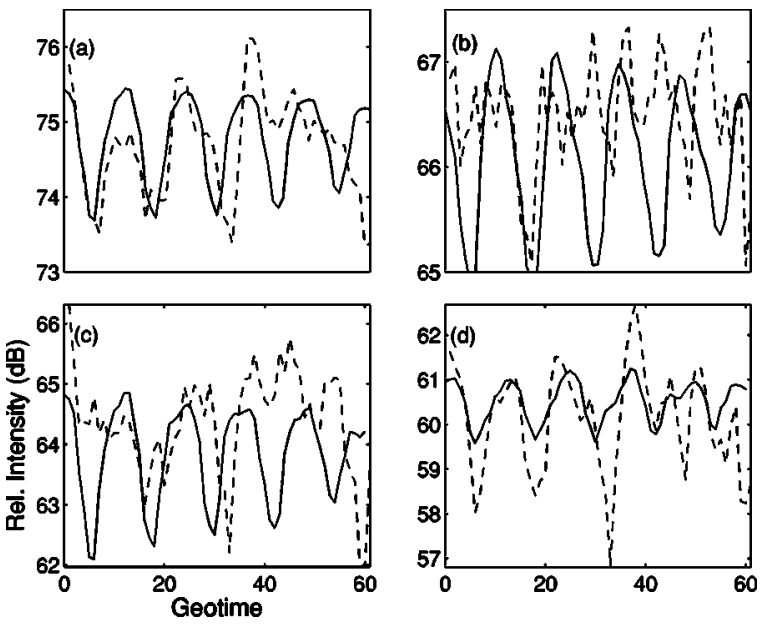

FIG. 12. Depth-averaged intensity variations for data (dashed curves) and PE simulations (solid curves): (a) full band, and Butterworth filtered bands centered at (b) $32 \mathrm{~Hz}$, (c) $64 \mathrm{~Hz}$, and (d) $95 \mathrm{~Hz}$. The relative intensity represents $I_{T}$ converted to $\mathrm{dB}$ re: $1 \mu \mathrm{Pa}$. Mean sound levels, quasiperiods, and amplitudes of variations are reproduced well by simulations for the full frequency band and two lower bands.

mum is modeled well in location and amplitude by computations. For higher frequency bands, the intensity data are cleaner. The $64 \mathrm{~Hz}$ band shows two intensity minima with locations and amplitudes that are produced by computations, and the $95 \mathrm{~Hz}$ band comparison emphasizes the accuracy of the peak locations. The amplitude variation of computations in the $95 \mathrm{~Hz}$ band is smaller than in the data, which is a likely reason for the somewhat reduced amplitude of the computed full band variations. Also, the average intensity levels of the computations are very similar to data.

The physical mechanism that is evidently responsible for producing the observed acoustic variations is well described in the literature. Resonant coupling between acoustic modes is caused by range-dependent anomalies in the bottom ${ }^{38}$ or water column ${ }^{7,11}$ when the following condition is satisfied:

$$
\kappa \approx k_{n}-k_{m},
$$

where $\kappa$ is the effective spectral peak wavenumber of the internal wave anomalies (in our situation $\kappa_{\mathrm{NRL}}$ ) and $k_{n}$ and $k_{m}$ are horizontal wavenumbers of acoustic modes $n$ and $m$. Equation (9) also arises because mode coupling occurs when acoustic wavenumber differences nearly coincide with peaks in the spectrum of inhomogeneities. ${ }^{16}$

Table II shows horizontal wavenumbers $k_{n}$ for the three lowest peak frequencies in the source spectrum. These wavenumbers were calculated using the program COUPLE ${ }^{39}$ for the bottom parameters shown in Fig. 7 and sound-speed profile calculated from an average of CTD measurements taken by the R/V Cape Hatteras. Table III shows differences
TABLE II. Acoustic wavenumbers calculated using COUPLE for the model environment at the source with no internal waves. Three, five, and seven propagating modes occur near 32, 64, and $95 \mathrm{~Hz}$.

\begin{tabular}{llll}
\hline \hline & $32 \mathrm{~Hz}$ & $64 \mathrm{~Hz}$ & $95 \mathrm{~Hz}$ \\
\hline 1 & 0.131 & 0.268 & 0.399 \\
2 & 0.117 & 0.258 & 0.391 \\
3 & 0.102 & 0.243 & 0.381 \\
4 & & 0.232 & 0.366 \\
5 & & 0.216 & 0.355 \\
6 & & & 0.343 \\
7 & & & 0.316 \\
\hline \hline
\end{tabular}

between selected wavenumbers from Table II. Specific wavenumber differences within $10 \%$ of effective internal wave wavenumber spectrum peaks (the fourth column of Table I) have been underlined. Numerous opportunities are available to satisfy resonance conditions at 32,64, and 95 Hz. The number of possible resonance interactions is the probable reason this particular packet configuration provides comparable variations in data and computations. Also, the resonant coupling opportunities for all three dominant acoustic frequencies is a likely reason for the similarity of $I_{T}$ variations seen in all panels of Fig. 12.

Two aspects of this analysis bear mentioning. First, the speed of the nonlinear internal wave packet is lower than estimates obtained from other SWARM data, ${ }^{40}$ but these estimates come from different days of the experiment. Second is the use of $45^{\circ}$ as the incidence angle, which is slightly smaller than the $49^{\circ}$ obtained from bearing estimates and experimental geometry. This variation can be acounted for by uncertainty in these estimates or by a slight curvature of the nonlinear internal wavefronts. Several combinations of internal wave parameters were tested with $\Lambda$ ranging from 180 to $350 \mathrm{~m}$ and $r_{n}$ from $2 n \Lambda$ to $2.5 n \Lambda$, but the formulation used above, with $\Lambda_{\mathrm{NRL}}=195 \mathrm{~m}, r_{n}=2.3 n \Lambda_{\mathrm{NRL}}$, and $v_{\mathrm{NRL}}=0.6 \mathrm{~m} / \mathrm{s}$, provided the best internal wave spectrum match. In addition, this configuration provided the largest acoustic variations and appropriate periods. This supports the hypothesis that the internal wave-acoustic resonance condition governs observed variations and a further analysis is presented in Sec. VI.

\section{PARAMETER SENSITIVITY}

According to the resonance condition in Eq. (9), mode coupling will be affected by changes to internal wave spectral peak locations, governed by internal wave parameters, or acoustic wavenumbers, which depend on water column and geoacoustic parameters. ${ }^{41}$ Ray theoretic intensity calculations $^{42}$ and scintillation index correlations ${ }^{6}$ also indicate

TABLE III. Differences between selected acoustic wavenumbers from Table II. Comparisons with wavenumbers $\kappa_{\mathrm{NRL}}$ in Table I show multiple opportunities for acoustic mode coupling within the internal wave packet. Underlined values are within $10 \%$ of a $\kappa_{\mathrm{NRL}}$ peak.

\begin{tabular}{lcccccccc}
\hline \hline & $k_{1}-k_{2}$ & $k_{1}-k_{3}$ & $k_{2}-k_{3}$ & $k_{2}-k_{4}$ & $k_{3}-k_{4}$ & $k_{1}-k_{5}$ & $k_{3}-k_{5}$ & $k_{4}-k_{6}$ \\
\hline $32 \mathrm{~Hz}$ & $\underline{0.0140}$ & 0.0283 & $\underline{0.0143}$ & & & & & \\
$64 \mathrm{~Hz}$ & 0.00928 & $\underline{0.0237}$ & $\underline{0.0144}$ & $\underline{0.0257}$ & 0.0113 & 0.0520 & $\underline{0.0271}$ & \\
$95 \mathrm{~Hz}$ & 0.00752 & 0.0183 & 0.0107 & $\underline{0.0258}$ & $\underline{0.0151}$ & $\underline{0.0443}$ & $\underline{0.0260}$ & $\underline{0.0230}$ \\
\hline \hline
\end{tabular}


acoustic sensitivity to nonlinear internal wave parameters. Despite the quantity of environmental data and the consistent modeling results, uncertainty in conclusions about internal wave speed, $v_{n}$, width $\Lambda_{n}$, and spacing $\Delta r_{n}$ in Eq. (8) are unavoidable. Limited knowledge of the water column and geoacoustic parameters also introduce ambiguity into computational results. Finally, the resonance condition itself cannot specify unique sets of nonlinear internal wave parameters. ${ }^{7}$

In this section, the dependence of acoustic variations on model parameters is described. In order to isolate resonance effects from range dependence, broadband $(10-180 \mathrm{~Hz})$ twodimensional (2-D) PE simulations are performed in a $71 \mathrm{~m}$ deep range-independent waveguide using the source model in Sec. IV and geoacoustic parameters in Fig. 7. The signals are received at 16 depths, from $15 \mathrm{~m}$ and every 3.5 to $69 \mathrm{~m}$. In all cases internal wave packets start $100 \mathrm{~m}$ from the source, have amplitudes $A_{n}=A=15 \mathrm{~m}$, and propagate at speed $v_{n}=v=0.65 \mathrm{~m} / \mathrm{s}$ toward the source for $30 \mathrm{~min}$. Except where otherwise noted, the packets consist of evenly spaced waves with $\Delta r_{n}=\Delta r$. The computational evidence shows that the resonance condition can predict increases in acoustic variability with geotime, indicating that the actual variations result from mode coupling. The soliton amplitude will not affect peak locations in a packet's normalized spectrum, so soliton amplitude effects are not discussed.

\section{A. Internal wave parameters}

For a packet of nonlinear internal waves with equal amplitudes, widths, and spacings, the number of waves $N>1$ does not affect the locations of the spectral peaks. Consequently, the occurrence of resonant mode coupling is not significantly affected. Geotime simulations performed that verify this assertion, for $N$ between 3 and 9 waves, show that the amplitudes of $I_{T}$ variations were always very similar. ${ }^{37}$ Unless noted otherwise, PE simulations here use $N$ $=5 \operatorname{sech}^{2}$ waves.

\section{Width}

In contrast to $N$, the width parameter $\Lambda$ does affect the Fourier spectrum of Eq. (8). In Sec. V, acoustic variability consistent with data was produced with $\Lambda=195 \mathrm{~m}$. Here nonlinear internal wave packets with larger widths are examined to determine if packet spectral peak locations can predict maxima in acoustic variability. Figure 13 shows normalized spectral peak behavior with $\Lambda$ between 300 and $800 \mathrm{~m}$ for a $\Delta r=2.3 \Lambda$ nonlinear internal wave packet. The frequency axis is calculated using a sampling rate of $1 / 30 \mathrm{~Hz}$. As the width $\Lambda$ of each waveform increases, the dominant frequency of the internal wave packet decreases. For smaller $\Lambda$, the first (lowest-frequency) peak is the maximum of the internal wave packet spectrum. The frequency location of the first peak and its spectral height decreases as $\Lambda$ increases. The location of the second peak also decreases with $\Lambda$, but its height increases until it becomes the spectral maximum. Further increases in $\Lambda$ emphasize lower-frequency components of the packet and cause more spectral peaks to occur at frequencies below the maximum peak location.

Acoustic wavenumber differences for 32, 64, and $95 \mathrm{~Hz}$

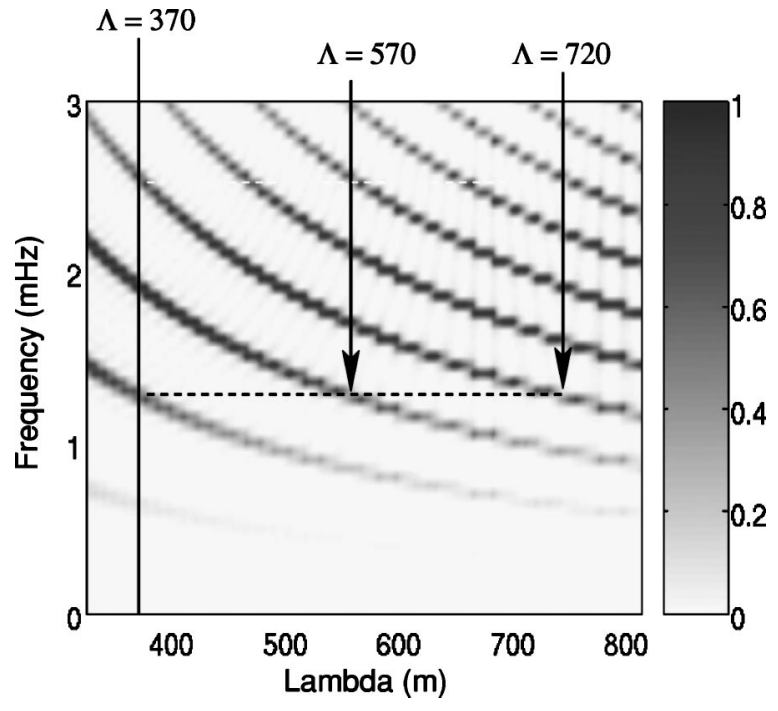

FIG. 13. Normalized internal wave spectrum for a packet with variable $\Lambda$ and $r_{n}=2.3 \Lambda$. As $\Lambda$ increases, dominant frequency components in the packet decrease. Resonance interactions occur for $\Lambda=370$, with peaks at 0.0014 and 0.0024 . Another set of peaks occur near these frequencies when $\Lambda \approx 570$ and $\Lambda \approx 720$.

are seen to correspond with internal wave packet frequencies near 0.0013 and $0.0024 \mathrm{~Hz}$ in Table I. By examining Fig. 13 we see that spectral peaks occur at these frequencies for several internal wave packets with larger $\Lambda$ than that used to model the NRL track. To confirm resonant behavior consistent with Eq. (9), we examine acoustic variations for internal wave packets with $\Lambda$ near $370 \mathrm{~m}$, which has spectral peaks near both 0.0013 and $0.0024 \mathrm{~Hz}$. Figure 14 shows results of broadband simulations for internal wave packets with five equally spaced values of $\Lambda$ from 325 to $425 \mathrm{~m}$, and Table IV shows $\mathrm{dB}$ amplitude values for $I_{T}$ fluctuations of full band geotime simulations and bandpass filtered simulations with center frequencies at 32,64, and $95 \mathrm{~Hz}$. As predicted, maximum variations occur for $\Lambda=375 \mathrm{~m}$, although $\Lambda=350 \mathrm{~m}$ also shows large-amplitude fluctuations, which can be seen in Figs. 14(b), 14(c), and in column 2 of Table IV. Amplitude degradation on either side of $\Lambda=375 \mathrm{~m}$ is evidence of a resonance peak for modal interaction. For the 32 and $64 \mathrm{~Hz}$ bands, $\Lambda=375 \mathrm{~m}$ causes maximum amplitude variations compared with $\Lambda=350 \mathrm{~m}$ in the $95 \mathrm{~Hz}$ band. This is consistent with Table III, which shows acoustic wavenumber differences occur for a slightly higher wavenumber (and thus a slightly higher frequency, near $0.0015 \mathrm{~Hz}$ ) in the $95 \mathrm{~Hz}$ band.

By following the internal wave spectral frequencies associated with resonance across Fig. 13 (black dashed lines) it appears that resonance points should also occur when $\Lambda$ is close to 570 and $720 \mathrm{~m}$. Table V shows an amplitude minimum at $\Lambda=675 \mathrm{~m}$ and larger variations for $\Lambda=575 \mathrm{~m}$ and $\Lambda=725 \mathrm{~m}$ that confirm the occurrence of resonant peaks. Table $\mathrm{V}$ indicates that 32 and $64 \mathrm{~Hz}$ mode coupling is prevalent for $\Lambda=575 \mathrm{~m}$, while $95 \mathrm{~Hz}$ coupling dominates variations caused by a packet with $\Lambda=725$. This is consistent with Table III, which shows two occurrences of $95 \mathrm{~Hz}$ band wavenumber differences with $0.026 \mathrm{rad} / \mathrm{m}$. Once again, this will occur at a slightly higher frequency than $0.0024 \mathrm{~Hz}$; also the $k_{1}-k_{3}$ value at $95 \mathrm{~Hz}$ is near $0.018 \mathrm{rad} / \mathrm{m}$, which corre- 


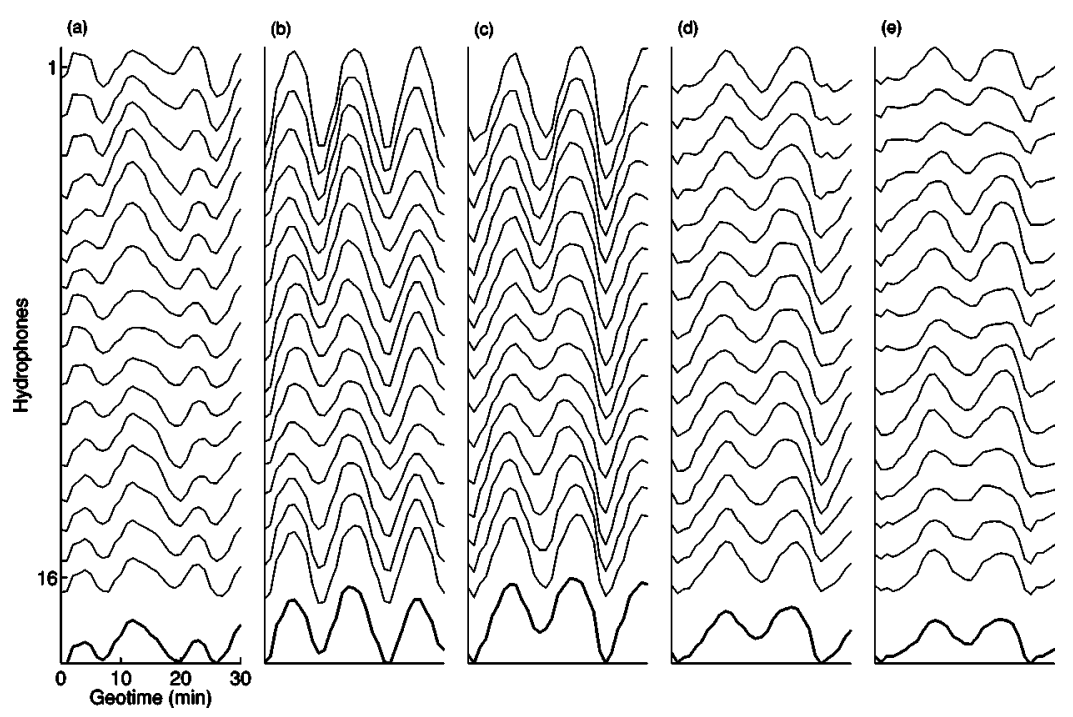

FIG. 14. Full band $I_{T}$ variations for different $\Lambda$ and $r_{n}=2.3 \Lambda$ as the nonlinear internal wave packet moves toward the source. (a) $\Lambda=325 \mathrm{~m}$, (b) $\Lambda=350 \mathrm{~m}$, (c) $\Lambda=375 \mathrm{~m}$, (d) $\Lambda=400 \mathrm{~m}$, and (e) $\Lambda=425 \mathrm{~m}$. All variations show a similar phase and weak depth dependence. The largest amplitudes occur in (b) and (c) with amplitude decay visible on either side.

sponds to the peak for $\Lambda=725 \mathrm{~m}$ just above the lower white dashed line in Fig. 13.

Together these two demonstrations show that nonlinear internal wave packet parameters can predict acoustic frequencies where large acoustic variability will occur. They also indicate that for a region without large packet spectral peaks at resonance frequencies, the acoustic variability will be significantly reduced.

\section{Spacing}

Spacing variations between individual sech-squared waves also affect the nonlinear internal wave packet spectrum. For evenly spaced packets, $\Delta r=s \Lambda$ where $s$ is called the spacing multiple. Due to the large acoustic variations observed (above), we focus here on internal wave packets with $\Lambda=375 \mathrm{~m}$. Figure 15 shows a normalized spectral peak dependence on $s$. As $s$ increases, the separation between internal wave peaks increases, decreasing the peak frequencies for the packet. Multiple spectral peaks arise for given $s$, and the dependence of the spectral maxima is similar to that in Fig. 13.

To illustrate resonant behavior, PE simulations are performed for broadband signals transmitted through nonlinear internal wave packets propagating toward the source with spacing multiples near 2.3. Table VI confirms the resonant behavior of $I_{T}$ fluctuation amplitudes. For full band signals, the largest amplitude occurs at $s=2.3$, with progressive amplitude decay for longer and shorter $s$. The $s=2.3$ case has spectral peak frequencies locations near 0.0014 and 0.0025 .

TABLE IV. $I_{T}$ variation amplitudes for different $\Lambda$ with $r_{n}=2.3 \Lambda$. Resonance effects expected near $\Lambda=375 \mathrm{~m}$. Amplitude maxima in 32 and $64 \mathrm{~Hz}$ bands for $\Lambda=375 \mathrm{~m}$, in a $95 \mathrm{~Hz}$ band for $\Lambda=350 \mathrm{~m}$.

\begin{tabular}{ccccc}
\hline \hline$\Lambda$ & Full & $32 \mathrm{~Hz}$ & $64 \mathrm{~Hz}$ & $95 \mathrm{~Hz}$ \\
\hline 325 & 1.27 & 0.89 & 2.73 & 1.71 \\
350 & 2.46 & 1.55 & 3.72 & 4.07 \\
375 & 2.78 & 3.07 & 5.10 & 3.30 \\
400 & 1.71 & 3.06 & 2.93 & 1.60 \\
425 & 1.31 & 1.60 & 1.86 & 1.82 \\
\hline \hline
\end{tabular}

Results centered at $95 \mathrm{~Hz}$ have maximum fluctuation amplitude for $s=2.15$, due to slightly higher wavenumber differences in this frequency band (Table III).

An examination of Fig. 15 reveals packet spectra peaks near $0.0014 \mathrm{~Hz}$ when $s \approx 4.3$ and near 0.0025 when $s \approx 3.6$. Table VII shows the amplitude of $I_{T}$ variations (in $\mathrm{dB}$ ) caused by packets with spacing multiples from 3.5 to 4.4 . For $10-180 \mathrm{~Hz}$ full band simulations, the amplitude peak at $s=3.6$ is followed by decay and then an increase to another peak at $s=4.4$. In addition, the $95 \mathrm{~Hz}$ band amplitude for $s$ $=4.2$ is relatively small given the resonance peak for $s$ $=4.3$. The small amplitude of depth-averaged oscillations at 64 and $95 \mathrm{~Hz}$ does not seem to support resonant condition behavior. However, the size of these depth-averaged values is affected by depth dependence of the pulse-averaged intensity curves. Figure 16 investigates bandpass filtered variations by a receiver for (a) $32 \mathrm{~Hz}$, (b) $64 \mathrm{~Hz}$, and (c) $95 \mathrm{~Hz}$. Very little depth dependence is present in Fig. 16(a), while Fig. 16(b) shows that near $20 \mathrm{~min}, I_{T}$ curves in the middle of the array are completely out of phase with curves near the top of the array. The variations thus tend to cancel each other out when depth averaging. Figure 16(c) shows largeamplitude fluctuations at each receiver, which is expected for $s=4.2$. Again strong depth dependence is present and oscillations change phase in alternating quarters of the water column. This depth dependence leads to a cancellation in depth-

TABLE V. $I_{T}$ variation amplitudes for different $\Lambda$ with $r_{n}=2.3 \Lambda$. Peak locations in Fig. 13 predict resonance interaction near $\Lambda=570 \mathrm{~m}$ and $\Lambda=720$. Column 2 shows $10-180 \mathrm{~Hz}$ full band simulations confirming resonant behavior. Interactions occur in all three frequency bands for $\Lambda=570 \mathrm{~m}$, and are restricted to 64 and $95 \mathrm{~Hz}$ bands for $\Lambda=720 \mathrm{~m}$.

\begin{tabular}{ccccc}
\hline \hline$\Lambda$ & Full & $32 \mathrm{~Hz}$ & $64 \mathrm{~Hz}$ & $95 \mathrm{~Hz}$ \\
\hline 550 & 2.08 & 1.78 & 4.66 & 3.36 \\
575 & 2.21 & 2.43 & 4.86 & 2.58 \\
600 & 1.70 & 2.40 & 3.46 & 1.56 \\
625 & 1.11 & 1.47 & 2.23 & 1.05 \\
650 & 1.23 & 1.08 & 1.94 & 1.48 \\
675 & 0.95 & 0.96 & 1.95 & 1.30 \\
700 & 1.64 & 0.98 & 1.84 & 2.71 \\
725 & 2.02 & 1.26 & 2.73 & 4.91 \\
\hline \hline
\end{tabular}




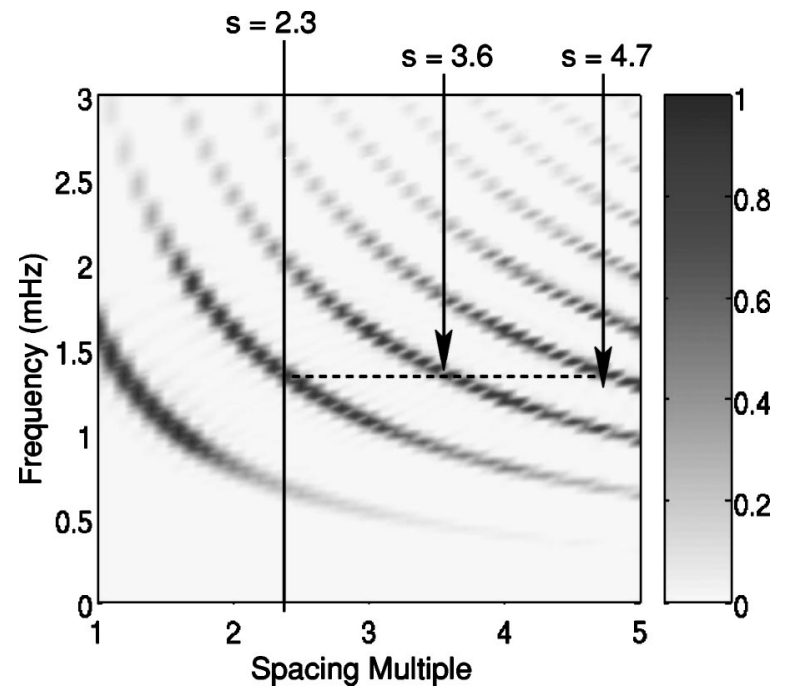

FIG. 15. Normalized internal wave spectrum dependence on spacing multiple $s$ with $\Lambda=370$. As $s$ increases, dominant frequency components in the packet decrease. Resonance interactions occur for $s=2.3$. Another set of peaks occur near $s=3.6$ and $s=4.2$.

averaged $I_{T}$ and explains the small amplitude of the $95 \mathrm{~Hz}$ band in Table VII

\section{B. Geoacoustic parameters}

A deep strong reflector can significantly impact wavenumbers and group speeds of low-frequency acoustic modes. ${ }^{41}$ Figure 17 shows sound speed, attenuation, and density profiles for nine bottom models. In model $R_{1}$ through $R_{9}$ the reflector increases in steps of $5 \mathrm{~m}$ from 6 to $46 \mathrm{~m}$ below the water-sediment interface, respectively. Model $R_{5}$ is similar to that used in Sec. IV. Changes in wavenumbers from varying reflector depths cause differences in relative mode arrival times in each frequency band. These changes can force the wavenumber difference to depart from internal wave spectral peak locations, resulting in less modal interaction and smaller $I_{T}$ variations.

Broadband simulations were performed using a nonlinear internal wave packet configuration with $\Lambda=370$ and $s$ $=2.3$. Figure 18 shows $I_{T}$ variations caused as the nonlinear internal wave packet moves toward the source for models $R_{3}$ through $R_{7}$. Large fluctuations occur at all hydrophones in Fig. 18(c), while significantly smaller-amplitude oscillations appear in the other panels. The variations in all cases are weakly depth dependent. Table VIII confirms that the $I_{T}$ fluctuation amplitude peak occurs with model $R_{5}$ for the full band and all filtered signals. The amplitude decrease associ-

TABLE VI. $I_{T}$ variation amplitudes for different $s$ with $\Lambda=370 \mathrm{~m}$. Resonance interactions most significant near $s=2.3$ for full-band, 32, and $64 \mathrm{~Hz}$ band simulations. The resonant peak occurs for $s=2.15$ in a $95 \mathrm{~Hz}$ band.

\begin{tabular}{lcccc}
\hline \hline \multicolumn{1}{c}{$s$} & Full & $32 \mathrm{~Hz}$ & $64 \mathrm{~Hz}$ & $95 \mathrm{~Hz}$ \\
\hline 2.0 & 1.36 & 0.93 & 2.76 & 2.03 \\
2.15 & 2.57 & 1.64 & 3.83 & 4.27 \\
2.3 & 2.78 & 3.07 & 5.11 & 3.30 \\
2.45 & 1.68 & 3.00 & 2.85 & 1.57 \\
2.6 & 1.27 & 1.54 & 1.72 & 1.84 \\
\hline \hline
\end{tabular}

TABLE VII. $I_{T}$ variation amplitudes for different $s$ with $\Lambda=370 \mathrm{~m}$. Resonance interactions are expected near $s=3.6$ and $s=4.1$. Full-band and $32 \mathrm{~Hz}$ band simulations show decay from resonant peak at $s=3.6$ but do not indicate a second peak. An amplitude increase occurs for 64 and $95 \mathrm{~Hz}$ bands.

\begin{tabular}{ccccc}
\hline \hline$s$ & Full & $32 \mathrm{~Hz}$ & $64 \mathrm{~Hz}$ & $95 \mathrm{~Hz}$ \\
\hline 3.5 & 1.86 & 1.92 & 5.28 & 1.64 \\
3.6 & 1.87 & 2.50 & 4.37 & 1.70 \\
3.7 & 1.59 & 2.26 & 3.13 & 1.66 \\
3.8 & 1.33 & 1.74 & 2.26 & 1.36 \\
3.9 & 1.14 & 1.32 & 1.57 & 1.49 \\
4.0 & 1.05 & 1.03 & 1.52 & 1.16 \\
4.1 & 1.08 & 0.90 & 1.90 & 1.21 \\
4.2 & 1.13 & 0.95 & 1.87 & 1.23 \\
4.3 & 1.45 & 0.93 & 2.01 & 2.36 \\
4.4 & 1.74 & 0.85 & 1.58 & 4.36 \\
\hline \hline
\end{tabular}

ated with raising and lowering the strong reflector from $26 \mathrm{~m}$ occurs because acoustic wavenumber differences for those configurations do not cause resonant interaction.

The effect of the shallow reflector was also considered. Acoustically hard material is characterized by higher sound speed, ${ }^{25}$ so decreasing the maximum sound speed of the shallow reflector could affect acoustic variability for several reasons. First, the amount of energy reflected back into the water column is reduced and energy is less effectively trapped between the shallow and deep reflectors. Second, reduced sound-speed gradients above and below the shallow reflector will also modify acoustic intensity. PE computations for several shallow sediment configurations were considered. The conclusion is that variations in the shallow reflector do not impact acoustic horizontal wavenumbers (and changes in $I_{T}$ amplitude) as significantly as the location of the deep reflector (for the lowest frequencies, the shallow reflector may even be acoustically transparent).

\section{Sensitivity estimation}

If an internal soliton with a particular width $\Lambda_{0}\left(f_{0}\right)$ defined by Eq. (9) causes resonant modal interaction across a
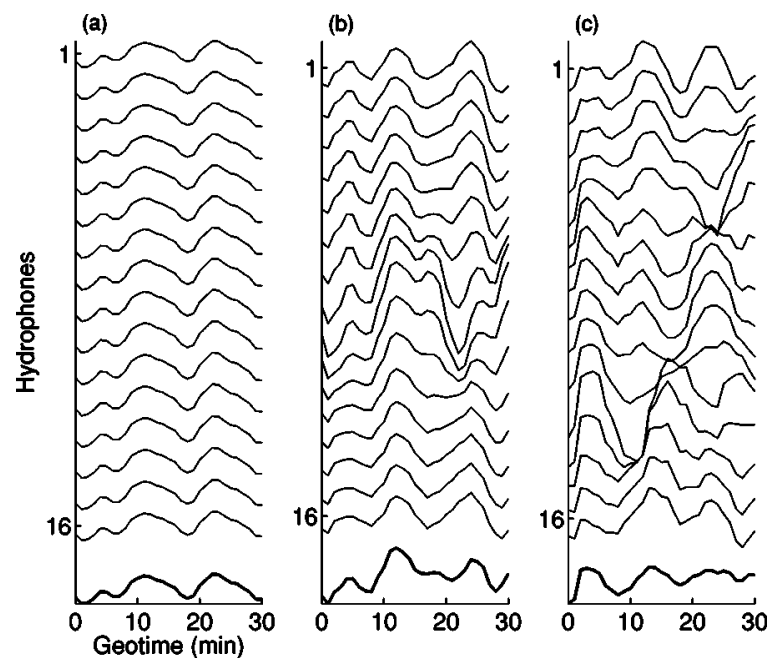

FIG. 16. Depth-dependent $I_{T}$ variations for $\Lambda=370 \mathrm{~m}$ and $s=4.2$ : (a) the 32 $\mathrm{Hz}$ band shows little depth dependence; (b) the $64 \mathrm{~Hz}$ band shows depth dependence in the middle phones beginning near $20 \mathrm{~min}$. (c) The $95 \mathrm{~Hz}$ band shows significant variations at each receiver. Depth-averaged variations in (c) show significant cancellation. 

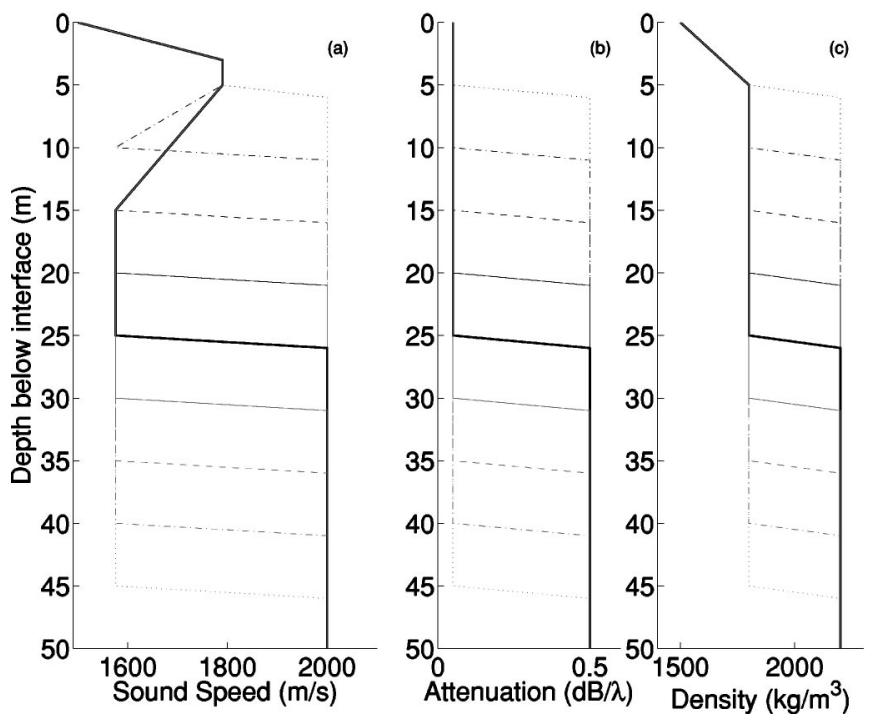

frequency band in oceanic waveguides, the associated internal wave spectral peak must have sufficient amplitude. The peak must also be broad enough to permit coupling for $\Lambda$ reasonably close to $\Lambda_{0}$, even though the amount of coupling may be reduced. A related matter is to estimate the bandwidth around $f_{0}$ that can be expected to exhibit variability due to mode coupling.

To address these issues, assume Eq. (9) holds for a resonant soliton width $\Lambda_{0}$ associated with acoustic modes $n$ and $m$ at radian frequency $\omega_{0}=2 \pi f_{0}$, where $k_{i}\left(\omega_{0}\right)$ is the $i$ th acoustic horizontal wavenumber, so

$$
\Lambda_{0}\left(\omega_{0}\right)=\frac{2 \pi}{k_{n}\left(\omega_{0}\right)-k_{m}\left(\omega_{0}\right)} .
$$

Is $\Lambda_{0}$ still resonant for an acoustic signal with frequency $\omega_{0}+\Delta \omega$ ? Expanding the wavenumber in a Taylor series for a band near $\omega_{0}$ and dropping higher-order terms gives

$$
k_{n}\left(\omega_{0}+\Delta \omega\right) \approx k_{n}\left(\omega_{0}\right)+k_{n}^{\prime}\left(\omega_{0}\right) \Delta \omega .
$$

Since
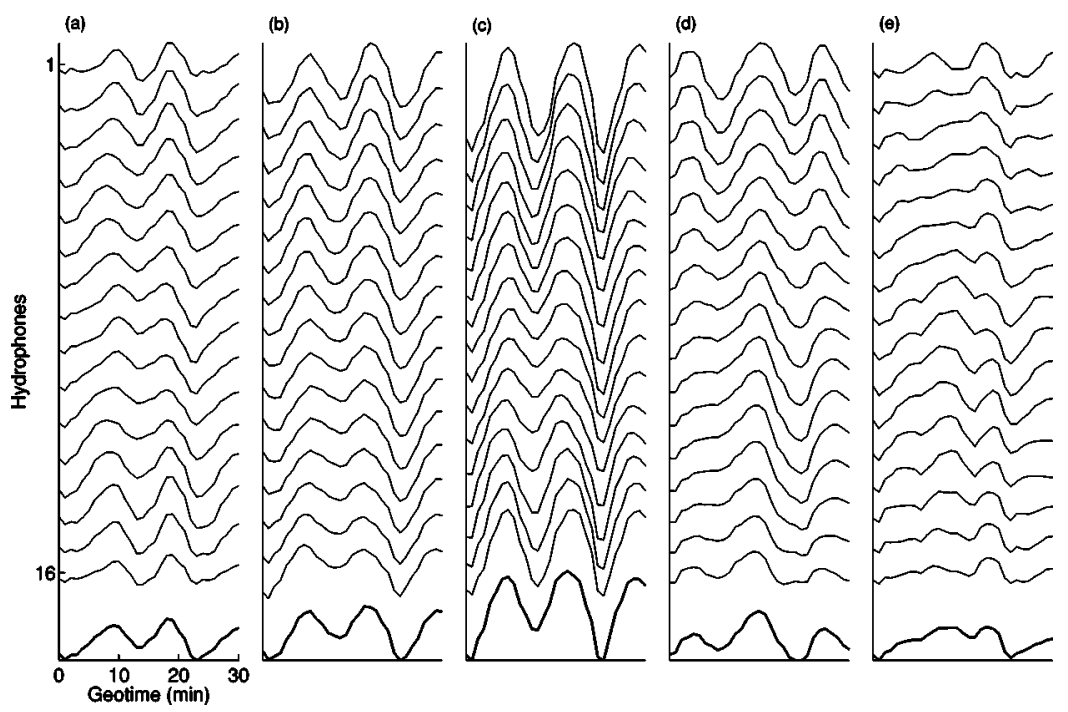

FIG. 17. (a) Sound speed $(\mathrm{m} / \mathrm{s})$, (b) attenuation $(\mathrm{dB} / \mathrm{\lambda})$, and (c) density $\left(\mathrm{kg} / \mathrm{m}^{3}\right)$ for geoacoustic models. Model $R_{1}$ (light dotted curve), $R_{2}$ (light dashed-dotted curve), $R_{3}$ (light dashed curve), $R_{4}$ (light solid curve), $R_{5}$ (heavy black curve), $R_{6}$ (black solid curve), $R_{7}$ (black dashed curve), $R_{8}$ (black dashed-dotted curve), and $R_{9}$ (black dotted curve) have a $2000 \mathrm{~m} / \mathrm{s}$ reflector at 6,11 , $16,21,26,31,36,41$, and $46 \mathrm{~m}$ from the watersediment interface, respectively.

$$
\begin{aligned}
k_{n}\left(\omega_{0}\right)-k_{m}\left(\omega_{0}\right) & =\omega_{0}\left[S_{p}^{n}\left(\omega_{0}\right)-S_{p}^{m}\left(\omega_{0}\right)\right] \\
& \equiv \omega_{0} \Delta S_{p}^{n m}\left(\omega_{0}\right),
\end{aligned}
$$

where $S_{p}^{i}(\omega)$ is the mode $i$ phase slowness, and

$$
k_{n}^{\prime}\left(\omega_{0}\right)-k_{m}^{\prime}\left(\omega_{0}\right)=\omega_{0}\left[S_{g}^{n}\left(\omega_{0}\right)-S_{g}^{m}\left(\omega_{0}\right)\right] \equiv \Delta S_{g}^{n m}\left(\omega_{0}\right),
$$

where $S_{g}^{i}(\omega)$ is the mode $i$ group slowness, Eq. (10) can be rewritten in terms of the Taylor series in Eq. (11) as

$$
\Lambda_{0}\left(\omega_{0}+\Delta \omega\right) \approx \frac{2 \pi}{\omega_{0} \Delta S_{p}^{n m}\left(\omega_{0}\right)+\Delta \omega \Delta S_{g}^{n m}\left(\omega_{0}\right)} .
$$

Thus, in order for $\Lambda_{0}$ to remain a resonant wavelength, any change in $\Delta S_{p}^{n m}\left(\omega_{0}\right)$ must be offset by a change in $\Delta S_{g}^{n m}\left(\omega_{0}\right)$ that is opposite in sign. This requirement is reasonable in shallow waveguides where increased frequency usually leads to reduced phase velocity and increased group velocity (past the Airy phase).

A useful example of Eq. (14) is a rigid-bottom isovelocity waveguide of sound speed $c$ and depth $D$, with wavenumbers for mode $n$ at frequency $f_{0}$,
FIG. 18. $I_{T}$ variations for different reflector depths using $\Lambda=370$ and $s=2.3$ for model (a) $R_{3}$, (b) $R_{4}$, (c) $R_{5}$, (d) $R_{6}$, and (e) $R_{7}$. All variations exhibit similar phase and weak depth dependence. Large-amplitude $I_{T}$ fluctuations in (c) and decreasing amplitudes on either side is evidence of a resonant interaction. 
TABLE VIII. $I_{T}$ variation amplitudes for different geoacoustic models. The resonance peak occurs as the reflector passes through $26 \mathrm{~m}$ depth in all frequency bands.

\begin{tabular}{ccccc}
\hline \hline Model & Full & $32 \mathrm{~Hz}$ & $64 \mathrm{~Hz}$ & $95 \mathrm{~Hz}$ \\
\hline$R_{1}$ & 0.84 & 1.20 & 1.26 & 1.35 \\
$R_{2}$ & 1.08 & 1.68 & 1.67 & 1.34 \\
$R_{3}$ & 1.23 & 1.65 & 1.93 & 1.15 \\
$R_{4}$ & 1.66 & 2.31 & 2.28 & 1.68 \\
$R_{5}$ & 2.95 & 2.82 & 5.00 & 3.91 \\
$R_{6}$ & 1.47 & 1.48 & 2.40 & 2.40 \\
$R_{7}$ & 1.00 & 1.32 & 1.71 & 1.46 \\
$R_{8}$ & 1.02 & 1.37 & 1.57 & 1.43 \\
$R_{9}$ & 1.12 & 1.02 & 1.56 & 1.39 \\
\hline \hline
\end{tabular}

$$
k_{n}(f)=\sqrt{\left(\frac{2 \pi f}{c}\right)^{2}-\left[\frac{\left(n-\frac{1}{2}\right) \pi}{D}\right]^{2}} .
$$

Equation (11) in terms of $f$ is

$$
k_{n}\left(f_{0}+\Delta f\right)=k_{n}\left(f_{0}\right)+\left(\frac{f_{0} \Delta f}{k_{n}\left(f_{0}\right)}\right)\left(\frac{2 \pi}{c}\right)^{2},
$$

and Eq. (14) becomes

$$
\Lambda_{0} \approx \frac{2 \pi}{k_{n}\left(f_{0}\right)-k_{m}\left(f_{0}\right)+\left(\frac{2 \pi k_{0} f_{0} \Delta f}{c}\right)\left(\frac{1}{k_{n}\left(f_{0}\right)}-\frac{1}{k_{m}\left(f_{0}\right)}\right)} .
$$

This relation only occurs if $\Delta f=0, m=n$, or $k_{m}\left(f_{0}\right)$ $=k_{n}\left(f_{0}\right)$. These are all trivial cases in the rigid bottom waveguide, which indicates that the resonant peaks are indeed sharp. However, other waveguides allow $k_{n}^{\prime}\left(f_{0}\right)$ $\approx k_{m}^{\prime}\left(f_{0}\right)$, which can occur asymptotically at high frequencies, at the Airy phase, or in multilayered waveguides. ${ }^{23}$

Using this example we also examine the change in optimal soliton width for a given variation $\Delta f$ from $f_{0}$ :

$$
\begin{aligned}
\Delta \Lambda= & \Lambda\left(f_{0}+\Delta f\right)-\Lambda\left(f_{0}\right) \\
= & \frac{2 \pi}{k_{n}\left(f_{0}+\Delta f\right)-k_{m}\left(f_{0}+\Delta f\right)}-\frac{2 \pi}{k_{n}\left(f_{0}\right)-k_{m}\left(f_{0}\right)} \\
= & \frac{2 \pi}{\underbrace{k_{n}\left(f_{0}\right)-k_{m}\left(f_{0}\right)}_{a}-\Delta f \underbrace{\left(\frac{4 \pi^{2} f_{0}}{c^{2} k_{n}\left(f_{0}\right)}-\frac{4 \pi^{2} f_{0}}{c^{2} k_{m}\left(f_{0}\right)}\right)}_{b}} \\
& -\frac{2 \pi}{k_{n}\left(f_{0}\right)-k_{m}\left(f_{0}\right)} \\
\approx & \frac{2 \pi b \Delta f}{a^{2}} .
\end{aligned}
$$

Large values of Eq. (18) indicate that the resonant soliton wavelength must undergo significant changes for small changes in frequency. If this happens, then a model soliton width would be unable to cause a modal interaction across a
TABLE IX. Variability in $\Delta \Lambda_{i j} / \Delta f$ for modes $i$ and $j$.

\begin{tabular}{lcccccccc}
\hline \hline & $\Delta \Lambda_{12}$ & $\Delta \Lambda_{13}$ & $\Delta \Lambda_{23}$ & $\Delta \Lambda_{24}$ & $\Delta \Lambda_{34}$ & $\Delta \Lambda_{15}$ & $\Delta \Lambda_{35}$ & $\Delta \Lambda_{46}$ \\
\hline $32 \mathrm{~Hz}$ & 14.5 & 9.8 & 6.2 & & & & & \\
$64 \mathrm{~Hz}$ & 13.6 & 4.7 & 7.2 & 3.0 & 5.2 & 1.7 & 2.5 & \\
$95 \mathrm{~Hz}$ & 13.5 & 4.6 & 6.9 & 2.8 & 4.8 & 1.4 & 2.1 & 1.7 \\
\hline \hline
\end{tabular}

frequency band, because Eq. (10) would not be satisfied. However, a relatively small value from Eq. (18) allows a soliton width selected for a particular center frequency to cause a modal interaction (though perhaps not at optimal resonance levels) over a frequency band $\Delta f$.

Table IX shows values of $\Delta \Lambda / \Delta f$ calculated using Eq. (18) for mode combinations shown in Table III. Row one shows changes near $10 \mathrm{~m} / \mathrm{Hz}$ for all mode combinations. For a $10 \mathrm{~Hz}$ bandwidth signal $\Delta f=5 \mathrm{~Hz}$ so $\Delta \Lambda \approx 50 \mathrm{~m}$-about $20 \%$ of a soliton width. At $64 \mathrm{~Hz}$, the largest value occurs for $\Delta \Lambda_{12}$. This value is about $50 \%$ of a typical wavelength, so it may be difficult to have interactions between these two modes. The other $64 \mathrm{~Hz}$ values are much smaller, especially for resonant mode combinations displayed in Table III, so interaction among these is feasible. Values of $\Delta \Lambda / \Delta f$ for 95 $\mathrm{Hz}$ follow the same pattern. These results are reasonable since Table IV and Table V demonstrate that for the $32 \mathrm{~Hz}$ band, significantly larger acoustic variability arises when $\Lambda$ is within $50 \mathrm{~m}$ of the soliton width that causes the largestamplitude variability. In the 64 and $95 \mathrm{~Hz}$ bands, the resonance peaks are sharper in Tables IV and V, which is confirmed by the reduced values for these frequency bands in Table IX. These estimates indicate the resonant soliton width is fairly sensitive to the acoustic frequency, especially in the lowest mode pairs, but the use of rigid bottom wavenumbers represents a worst-case scenario for the sensitivity. The values in Table IX are also consistent with results for nonlinear internal wave packet randomness since variability of about 20-30 $\mathrm{m}$ in $\Lambda_{i w}$ do not significantly affect the internal wave spectrum.

Table $\mathrm{X}$ shows values of $\Delta \Lambda / \Delta D$ that result from a perturbation analysis similar to that for $\Delta \Lambda / \Delta f$. These values were calculated for $32 \mathrm{~Hz}$ using $D=96$, and for the higher frequencies using $D=71$. This difference is necessary to make isospeed wavenumbers correspond more closely to wavenumbers in Table I, and is expected since the $32 \mathrm{~Hz}$ band penetrates to the deep reflector while the higherfrequency modes are more effectively contained in the water column. Values in Table $\mathrm{X}$ are significantly larger than those obtained for $\Delta \Lambda / \Delta f$, which is consistent with the conclusion from Sec. VIB that resonant modal interaction is relatively sensitive to waveguide and deep reflector depths.

TABLE X. Variability in $\Delta \Lambda_{i j} / \Delta D$ for modes $i$ and $j$.

\begin{tabular}{rrrrrrrrr}
\hline \hline & $\Delta \Lambda_{12}$ & $\Delta \Lambda_{13}$ & $\Delta \Lambda_{23}$ & $\Delta \Lambda_{24}$ & $\Delta \Lambda_{34}$ & $\Delta \Lambda_{15}$ & $\Delta \Lambda_{35}$ & $\Delta \Lambda_{46}$ \\
\hline $32 \mathrm{~Hz}$ & 16.4 & 5.5 & 8.3 & & & & & \\
$64 \mathrm{~Hz}$ & 24.2 & 8.1 & 12.1 & 4.9 & 8.2 & 2.5 & 4.7 & \\
$95 \mathrm{~Hz}$ & 35.9 & 12.0 & 18.0 & 7.2 & 12.0 & 3.6 & 5.1 & 4.1 \\
\hline \hline
\end{tabular}




\section{SUMMARY AND DISCUSSION}

An environmental model for the NRL acoustic track is formulated using thermistor data at the receiver, CTD data at the source, and perturbations of AMCOR 6010 borehole data. Due to the apparent contamination in the source monitor signal, a model source signature is developed for broadband acoustic modeling. Nonlinear internal wave packets that were observed between the acoustic source and receiver during transmission are modeled using a train of $\mathrm{sech}^{2}$ solitons. This model allows the soliton width and spacing between solitons to be varied for each wave in the packet.

It has been found by comparing broadband SWARM acoustic data to broadband PE computations that the passage of nonlinear internal waves evidently cause the pulseaveraged intensity fluctuations in airgun signals recorded at the NRL/VLA. Wavelet transforms are used to analyze time-frequency variations of acoustic data caused by nonlinear internal waves. The transforms reveal that acoustic mode strength and arrival time variability of peak frequency components are correlated with the passage of the nonlinear internal waves.

The acoustic frequency bands near 32, 64, and $95 \mathrm{~Hz}$ studied here are significantly lower frequencies than have been previously examined in connection with nonlinear internal waves. While the internal wave-acoustic resonance condition is well known, the opportunity to relate observed nonlinear internal wave spectral peaks to acoustic wavenumbers is apparently novel. Significant acoustic effects arise despite the relatively low number of propagating modes at the frequencies of interest. The influence of nonlinear internal wave width and spacing parameters on acoustic variability is described, and the internal wave spectrum can predict packet configurations that cause resonant behavior for particular acoustic frequency bands.

Several aspects of the acoustic results from the NRL track remain open for future investigation. Time-frequency behavior observed in data and simulation scalograms was not fully analyzed and would be a rich area of investigation. New applications of time-frequency analysis, including inversions, may be possible with an understanding of the nonlinear internal wave effects. Range-dependent inversions along the NRL acoustic track could be performed for bottom or water-column properties. The influence of uncertainty modeled by random internal wave parameters deserves more extensive treatment.

\section{ACKNOWLEDGMENTS}

The authors would like to thank Yongke $\mathrm{Mu}$ for assistance reading the acoustic data and Steve Forsythe for insightful discussions involving signal processing related to the source monitor. This work was supported by an ONR Ocean Acoustics Graduate Traineeship Award and by ONR grants to Rensselaer, the University of Delaware, and Woods Hole Oceanographic Institution. This is WHOI contribution No. 11257.

${ }^{1}$ J. Zhou, X. Zhang, and P. H. Rogers, "Resonant interaction of sound waves with internal solitons in the coastal zone," J. Acoust. Soc. Am. 90, 2042-2054 (1991).
${ }^{2}$ C. O. Tiemann, P. F. Worcester, and B. D. Cornuelle, “Acoustic scattering by internal solitary waves in the Strait of Gibraltar," J. Acoust. Soc. Am. 109, 143-154 (2001).

${ }^{3}$ D. Rubenstein and M. H. Brill, "Acoustic variability due to internal waves and surface waves in shallow water," in Ocean Variability and Acoustic Propagation, edited by J. Potter and A. Warn-Varnas (Kluwer Academic, Boston, 1991), pp. 215-228.

${ }^{4}$ A. K. Liu, "Analysis of nonlinear waves in the New York Bight," J. Geophys. Res. 93, 12317-12329 (1988).

${ }^{5}$ J. F. Lynch, G. Jin, R. Pawlowicz, D. Ray, A. J. Plueddermann, C.-S. Chiu, J. H. Miller, R. H. Bourke, A. R. Parsons, and R. Muench, "Acoustic travel-time perturbations due to shallow-water internal waves and internal tides in the Barents Sea Polar Front: Theory and experiment," J. Acoust. Soc. Am. 99, 803-821 (1996).

${ }^{6}$ D. Rubenstein, "Observations of cnoidal internal waves and their effect on acoustic propagation in shallow water," IEEE J. Ocean. Eng. 24, 346357 (1999).

${ }^{7}$ L. B. Dozier and F. D. Tappert, "Statistics of normal mode amplitudes in a random ocean. I. Theory," J. Acoust. Soc. Am. 63, 353-365 (1978).

${ }^{8}$ X. Tang and F. D. Tappert, "Effects of internal waves on sound pulse propagation in the Straights of Florida," IEEE J. Ocean. Eng. 33, 245-255 (1997).

${ }^{9}$ J. C. Preisig and T. F. Duda, "Coupled acoustic mode propagation through continental-shelf internal solitary waves," IEEE J. Ocean. Eng. 22, 256269 (1997).

${ }^{10}$ T. F. Duda and J. C. Preisig, "A modeling study of acoustic propagation through moving shallow water solitary wave packets," IEEE J. Ocean. Eng. 24, 16-32 (1999).

${ }^{11}$ S. A. Ching-Bing, A. Warn-Varnas, D. B. King, K. G. Lamb, M. Teixeira, and J. A. Hawkins, "Analysis of coupled oceanographic and acoustic soliton simulations in the Yellow Sea: a search for soliton-induced resonances," Math. Comput. Simul. 62, 11-20 (2003).

${ }^{12}$ R. H. Headrick, J. F. Lynch, J. N. Kemp, A. E. Newhall, K. von der Heydt, J. R. Apel, M. Badiey, C.-S. Chiu, S. Finette, M. H. Orr, B. Pasewark, A. Turgut, S. N. Wolf, and D. Tielbuerger, "Modeling mode arrivals in the 1995 SWARM experiment acoustic transmissions," J. Acoust. Soc. Am. 107, 221-236 (2000).

${ }^{13}$ D. Rouseff, A. Turgut, S. N. Wolf, S. Finette, M. H. Orr, B. H. Pasewark, J. R. Apel, M. Badiey, C.-S. Chiu, R. H. Headrick, J. F. Lynch, J. N. Kemp, A. E. Newhall, K. von der Heydt, and D. Tielbuerger, "Coherence of acoustic modes propagating through shallow water internal waves," J. Acoust. Soc. Am. 111, 1655-1666 (2002).

${ }^{14}$ D. Tielbuerger, S. Finette, and S. N. Wolf, "Acoustic propagation through an internal wave field in a shallow water waveguide," J. Acoust. Soc. Am. 101, 789-808 (1997).

${ }^{15}$ J. F. Lynch, M. H. Orr, and S. N. Wolf, "Low frequency acoustic propagation through shallow water internal waves," in Sound Propagation Through Internal Waves (unpublished).

${ }^{16}$ B. G. Katsnel'son and S. A. Pereselkov, "Resonance effects in sound scattering by internal wave packets in a shallow sea," Acoust. Phys. 44, 684-689 (1998).

${ }^{17}$ J. R. Apel, M. Badiey, C.-S. Chiu, S. Finette, R. H. Headrick, J. Kemp, J. F. Lynch, A. E. Newhall, M. H. Orr, B. H. Pasewark, D. Tielbuerger, A. Turgut, K. von der Heydt, and S. N. Wolf, "An overview of the 1995 SWARM shallow-water internal wave acoustic scattering experiment," IEEE J. Ocean. Eng. 22, 465-499 (1996).

${ }^{18}$ M. Badiey, Y. Mu, J. F. Lynch, J. R. Apel, and S. N. Wolf, "Temporal and azimuthal dependence of sound propagation in shallow water with internal waves," IEEE J. Ocean. Eng. 27, 117-129 (2002).

${ }^{19}$ F. S. Henyey and A. Hoering, "Energetics of nonlinear internal waves," IEEE J. Ocean. Eng. 102, 3323-3330 (1999).

${ }^{20}$ S. Mallat, A Wavelet Tour of Signal Processing, 2nd ed. (Academic, San Diego, 1999).

${ }^{21}$ M. Badiey, I. Jaya, and A. H.-D. Cheng, "Shallow water acoustic/ geoacoustic experiments at the New Jersey Atlantic Generating Station site," J. Acoust. Soc. Am. 96, 3593-3604 (1994).

${ }^{22}$ G. R. Potty, J. H. Miller, J. F. Lynch, and K. B. Smith, "Tomographic inversion for sediment parameters in shallow water," J. Acoust. Soc. Am. 108, 973-986 (2000).

${ }^{23}$ I. Tolstoy and C. S. Clay, Ocean Acoustics: Theory and Experiment in Underwater Sound (American Institute of Physics, Woodbury, NY, 1966).

${ }^{24}$ M. D. Collins, "RAMGEO 1.5," URL ftp://albacore.nrl.navy.mil/RAM.

${ }^{25}$ F. B. Jensen, W. A. Kuperman, M. B. Porter, and H. Schmidt, Computa- 
tional Ocean Acoustics (Springer-Verlag, New York, 2000).

${ }^{26}$ S. Finette, M. H. Orr, A. Turgut, J. R. Apel, M. Badiey, C.-S. Chiu, R. H. Headrick, J. N. Kemp, J. F. Lynch, A. E. Newhall, K. von der Heydt, B. Pasewark, S. N. Wolf, and D. Tielbuerger, "Acoustic field variability induced by time evolving internal wave fields," J. Acoust. Soc. Am. 108, 957-972 (2000).

${ }^{27}$ R. Guenther, Modern Optics (Wiley, New York, 1990).

${ }^{28}$ T. C. Yang and K. Yoo, "Internal wave spectrum in shallow water: Measurement and comparison with the Garrett-Munk model," IEEE J. Ocean. Eng. 24, 333-345 (1999).

${ }^{29}$ B. J. Sperry, J. F. Lynch, G. Gawarkiewicz, C.-S. Chiu, and A. Newhall, "Characteristics of acoustic propagation to the eastern vertical line array receiver during the summer 1996 New England shelfbreak PRIMER experiment," IEEE J. Ocean. Eng. 28, 729-749 (2003).

${ }^{30}$ National Geophysical Data Center, NOAA, Hydrographic Survey Data, Vol. 1, Version 3.3.

${ }^{31}$ J. D. Milliman, A. Jiezao, L. Anchun, and J. I. Ewing, "Late quaternary sedimentation on the outer and middle New Jersey Continental shelf: Result of two local deglaciations?" J. Geol. 98, 966-976 (1990).

${ }^{32}$ S. D. Stearns, Digital Signal Analysis (Hayden, Rochelle Park, NJ, 1975).

${ }^{33}$ R. J. Cederberg, W. L. Siegmann, and W. M. Carey, "Influence of geoacoustic modeling on predictability of low-frequency propagation in rangedependent shallow-water environments," J. Acoust. Soc. Am. 97, 27542766 (1995).

${ }^{34}$ I. Rozenfeld, W. M. Carey, P. G. Cable, and W. L. Siegmann, "Modeling and analysis of sound transmission in the Strait of Korea," IEEE J. Ocean. Eng. 26, 809-819 (2001).

${ }^{35}$ A. Turgut and S. N. Wolf, "Matched-field inversion of seabed geoacoustic properties complemented by chirp sonar surveys," J. Acoust. Soc. Am. 110, 2661(A) (2001).

${ }^{36} \mathrm{Z}$. Y. Zhang and C. T. Tindle, "Improved equivalent fluid approximations for a low shear speed ocean bottom," J. Acoust. Soc. Am. 98, 3391-3396 (1995).

${ }^{37}$ S. D. Frank, "Modeling nonlinear internal wave effects on broadband shallow water acoustics," Ph.D. thesis, Rensselaer Polytechnic Institute, 2003.

${ }^{38}$ S. T. McDaniel and D. F. McCammon, "Mode coupling and the environmental sensitivity of shallow-water propagation loss," J. Acoust. Soc. Am. 82, 217-223 (1987).

${ }^{39}$ R. B. Evans, "A coupled mode solution for acoustic propagation in a waveguide with stepwise depth variations of a penetrable bottom," J. Acoust. Soc. Am. 74, 188-195 (1983).

${ }^{40}$ R. H. Headrick, J. F. Lynch, J. N. Kemp, A. E. Newhall, K. von der Heydt, J. R. Apel, M. Badiey, C.-S. Chiu, S. Finette, M. H. Orr, B. Pasewark, A. Turgut, S. N. Wolf, and D. Tielbuerger, "Acoustic normal mode fluctuation statistics in the 1995 SWARM internal wave scattering experiment," J. Acoust. Soc. Am. 107, 201-220 (2000).

${ }^{41}$ R. J. Cederberg, W. L. Siegmann, and M. J. Jacobson, "Predictability of acoustic intensity and horizontal wave numbers in shallow water at low frequencies using parabolic approximations," J. Acoust. Soc. Am. 94, 1034-1043 (1993).

${ }^{42}$ L. Baxter II and M. H. Orr, "Fluctuations in sound transmission through internal waves associated with the thermocline: A computer model for acoustic transmission through sound velocity fields calculated from thermistor chain, CTD, XBT, and acoustic backscattering," J. Acoust. Soc. Am. 71, 61-66 (1982). 